\title{
The Soft X-ray Properties of Nearby Low-Luminosity Active Galactic Nuclei and their Contribution to the Cosmic X-ray Background
}

\author{
Eve L. Halderson, Edward C. Moran ${ }^{1}$, Alexei V. Filippenko \\ Department of Astronomy, University of California, Berkeley, CA 94720-3411 \\ Luis C. Ho \\ The Observatories of the Carnegie Institution of Washington, 813 Santa Barbara St., Pasadena, \\ CA 91101
}

\begin{abstract}
We have examined ROSAT soft X-ray observations of a complete, distance-limited sample of Seyfert and LINER galaxies. X-ray data are available for 46 out of 60 such objects which lie within a hemisphere of radius $18 \mathrm{Mpc}$. We have constructed radial profiles of the nuclear sources in order to characterize their spatial extent and, in some cases, to help constrain the amount of flux associated with a nuclear point source. PSPC data from ROSAT have been used to explore the spectral characteristics of the objects with sufficient numbers of detected counts. Based on the typical spectral parameters of these sources, we have estimated the luminosities of the weaker sources in the sample. We then explore the relationship between the soft X-ray and $\mathrm{H} \alpha$ luminosities of the observed objects; these quantities are correlated for higher-luminosity AGNs. We find a weak correlation at low luminosities as well, and have used this relationship to predict $L_{\mathrm{X}}$ for the 14 objects in our sample that lack X-ray data. Using the results of the spatial and spectral analyses, we have compared the X-ray properties of Seyferts and LINERs, finding no striking differences between the two classes of objects. However, both types of objects often exhibit significant amounts of extended emission, which could minimize the appearance of differences in their nuclear properties. The soft X-ray characteristics of the type 1 and type 2 active galaxies in the sample are also discussed. We then compute the local X-ray volume emissivity of low-luminosity Seyferts and LINERs and investigate their contribution to the cosmic X-ray background. The $0.5-2.0 \mathrm{keV}$ volume emissivity of $2.2 \times 10^{38} \mathrm{ergs} \mathrm{s}^{-1} \mathrm{Mpc}^{-3}$ we obtain for our sample suggests that lowluminosity AGNs produce at least $9 \%$ of the soft X-ray background.
\end{abstract}

Subject headings: galaxies: active — galaxies: nuclei — galaxies: Seyfert — X-rays: galaxies

\footnotetext{
${ }^{1}$ Chandra Fellow.
} 


\section{Introduction}

The bolometric luminosities of active galactic nuclei (AGNs) span more than eight orders of magnitude from the most powerful quasars to the weakest known Seyfert galaxies. Despite their luminosity differences, however, quasars and Seyferts possess many of the same characteristics. In particular, their rest-frame optical spectra are remarkably similar, exhibiting a luminosity-independent relationship between the emission-line and nonstellar continuum strengths (Yee 1980; Shuder 1981; Ho \& Peng 2001). Thus, it has been suspected for some time that nearby, low-luminosity Seyfert nuclei and bright, distant quasars are powered by the same fundamental mechanism-accretion of matter onto a supermassive black hole.

The questions facing us now are whether there is a lower luminosity limit below which this mechanism does not operate, and how prevalent this type of nuclear activity is among galaxies today. In this context, the nature of the processes that power low-ionization nuclear emission-line regions (LINERs; Heckman 1980), which are found in a significant fraction of all nearby galaxies (Ho, Filippenko, \& Sargent 1997b), is of particular importance. Compared to Seyferts, LINERs display a lower degree of ionization in their emission-line spectra that could result from photoionization by a dilute nonstellar continuum associated with an AGN (Halpern \& Steiner 1983; Ferland \& Netzer 1983; Ho, Filippenko, \& Sargent 1993). However, other astrophysical processes are capable of producing LINER-like nebular spectra, including shocks from supernova explosions (Heckman 1980; Dopita \& Sutherland 1995) and photoionization by extremely hot stars in compact starbursts (Filippenko \& Terlevich 1992; Shields 1992; Barth \& Shields 2000). It may well be that LINERs are a heterogeneous class whose optical emission lines are powered in some cases by accretion and in others by stellar processes (e.g., Filippenko 1996). Determination of the fraction of LINERs that contain genuine active nuclei is needed to refine estimates of the incidence of supermassive black holes in galactic nuclei, an issue which is central to our understanding of galaxy formation and evolution. It is also vital for establishing the shape of the faint end of the AGN luminosity function, and in turn, the total contribution of AGNs to the extragalactic X-ray background (Comastri et al. 1995).

Observations of the luminosity, spectrum, and spatial extent of X-ray emission in galaxies are particularly useful for addressing the nature of their nuclear activity. For example, the soft X-ray emission of star-forming galaxies often includes thermal components that are extended on kiloparsec scales, whereas AGNs are typically associated with unresolved, nonthermal X-ray sources. Detection of a point-like X-ray source in the nucleus of M81 using the Einstein High Resolution Imager (Elvis \& van Speybroeck 1982) played a key role in establishing the presence of a weak AGN in this nearby object. As the first AGN to be identified with a soft X-ray luminosity of $\sim 10^{40}$ ergs $\mathrm{s}^{-1}$, this discovery extended the AGN luminosity function two orders of magnitude below the previously achieved limit. More recently, Koratkar et al. (1995) have investigated five other lowluminosity AGNs (LLAGNs) with ROSAT, finding that they resemble classical broad-line Seyfert galaxies in terms of the spatial extent and spectral characteristics of their nuclear X-ray emission. We might expect, therefore, that a careful comparison of the X-ray properties of LINERs and low- 
luminosity Seyfert galaxies would allow us to test for a common origin of the nuclear activity in the two types of objects.

Soft X-ray data are also useful for the investigation of AGN unification. It has been suggested that radio-quiet AGNs with and without broad optical emission lines (type 1 and type 2, respectively) are intrinsically similar objects that appear to be different because of the presence or absence of dense circumnuclear material along our line of sight (Antonucci 1993). The material thought to be obscuring the broad emission-line region and the optical/UV continuum source in type 2 AGNs should absorb a significant fraction of their soft X-ray fluxes as well (Mulchaey et al. 1993). Thus, a soft X-ray survey of a well-defined sample of AGNs containing both type 1 and type 2 objects might provide insight as to whether or not the unified model applies to the majority of AGNs.

To explore these issues, we have assembled a relatively unbiased, distance-limited sample of low-luminosity Seyferts and LINERs. The majority have been observed by ROSAT in the soft (0.1-2.4 keV) X-ray band. In the next two sections, we describe the sample of objects and the available data. In $\S 4$, we examine the spatial properties of the nuclear X-ray sources and present an analysis of their soft X-ray fluxes and luminosities. A comparison of our results for the various subclasses of objects represented in our sample is discussed in $\S 5$, along with our investigation of the LLAGN contribution to the soft X-ray background.

\section{A Distance-Limited LLAGN Sample}

Between 1984 and 1990, Ho, Filippenko, \& Sargent (1995, 1997a, 1997b; Ho et al. 1997c; see also Filippenko \& Sargent 1985) carried out an optical spectroscopic survey of nearby galactic nuclei in the northern sky. Galaxies with total apparent blue magnitudes $B_{\mathrm{T}}<12.5$ were included in the survey, which is nearly complete for $B_{\mathrm{T}}<12.0$ mag. Most (418) of the 486 objects observed have emission-line spectra indicative of some sort of nuclear activity (Ho et al. 1997b): 49\% of the emission-line nuclei are classified as star-forming (H II) galaxies, $23 \%$ are LINERs, $16 \%$ have "transition" spectra, which may represent a combination of H II and LINER characteristics, and the remainder $(12 \%)$ are Seyfert galaxies of various types.

Within a distance of $18 \mathrm{Mpc}, 60$ galaxies from the Ho et al. survey are classified as Seyferts (22) or LINERs (38). The names, spectroscopic classifications, distances, ${ }^{2}$ and $\mathrm{H} \alpha$ luminosities of these objects (from Ho et al. 1997a) are listed in Table 1. Only one galaxy, NGC 1068, has an H $\alpha$ luminosity substantially greater than $10^{40} \mathrm{ergs} \mathrm{s}^{-1}$; as Ho et al. (1997a) have discussed, this limit is useful for distinguishing high- and low-luminosity AGNs. Thus, since the Ho et al. survey is expected to be complete for nucleated galaxies out to $18 \mathrm{Mpc}$, the 60 Seyferts and LINERs in our

\footnotetext{
${ }^{2}$ Distances from Ho et al. (1997a) assume $H_{0}=75 \mathrm{~km} \mathrm{~s}^{-1} \mathrm{Mpc}^{-1}$, which we adopt throughout this paper. The distributions of distances for Seyferts and LINERs and for type 1 and type 2 objects are extremely similar, so we may compare different classes of objects without concern for systematic differences in distances.
} 
list constitute a large, relatively unbiased sample of low-luminosity AGNs and AGN candidates. ${ }^{3}$

\section{Soft X-ray Observations}

The majority of the objects in our sample have been observed with the ROSAT Observatory, using the High Resolution Imager (HRI) and the Position Sensitive Proportional Counter (PSPC) instruments on board. Both instruments are sensitive in an energy range of approximately 0.1-2.4 $\mathrm{keV}$. The HRI provides an angular resolution (half-energy width) of about $5^{\prime \prime}$ on-axis, but has very little spectral sensitivity. Its field of view is $38^{\prime}$ square, most of which is usable. The PSPC has lower angular resolution $\left(25^{\prime \prime}\right.$ at $\left.1 \mathrm{keV}\right)$, but it has modest spectral sensitivity, with an energy resolution $E / \Delta E=0.43(E / 0.93)^{-0.5}$. The full PSPC field of view covers a radius of $1^{\circ}$; however, vignetting and broadening of the point-response function degrade the image quality at off-axis angles greater than $\sim 10^{\prime}$ (see the ROSAT Mission Description, Appendix F).

ROSAT images of 44 of the 60 objects in our sample are available. We acquired these data through HEASARC at NASA's Goddard Space Flight Center. A total of 21 galaxies were observed with both the PSPC and HRI instruments. Another 15 objects were observed only with the PSPC, and 8 were observed with the HRI only. Two galaxies that were not observed by ROSAT, NGC 4698 and NGC 4762, were detected with the Einstein Imaging Proportional Counter (IPC) in the 0.2-3.5 $\mathrm{keV}$ band (Fabbiano, Kim, \& Trinchieri 1992). Thus, the majority of our sample (46 galaxies, or $77 \%$ ) has some type of soft X-ray data available.

For most of the galaxies, no more than one observation per ROSAT instrument was made. A handful of objects, however, were observed multiple times, often for monitoring purposes. We combined multiple images of a galaxy when its position in the field was the same. Otherwise, a single image providing both the longest individual exposure time and smallest off-axis angle was analyzed, except when the galaxy was partially obscured by one of the PSPC window support ribs. In such cases, an alternate image providing an unobstructed view of the object was used.

Exposure times range from $5 \mathrm{ks}$ to $115 \mathrm{ks}$ for the 29 HRI observations, and in each the galaxy is located less than $6^{\prime}$ off-axis. Source counts were extracted within a circular region large enough to include all of the flux arising near the nucleus $\left(20^{\prime \prime}-40^{\prime \prime}\right.$ in radius for point-like sources). A background count rate was determined in a concentric annulus. Contaminating sources in the background region were excluded, and the size of the annulus was chosen to ensure that at least 100 background counts were collected. All but six HRI sources were detected; $3 \sigma$ upper limits were computed for the non-detections. The majority of the 36 galaxies imaged with the PSPC are located within $15^{\prime}$ of the field center, although we analyzed data for some objects observed as much

\footnotetext{
${ }^{3}$ The nature of the nuclear activity in the transition objects is still under investigation, so we have omitted them from the present study. See Ho \& Ulvestad (2001, Appendix A) for a discussion of issues concerning completeness and selection biases in AGN samples, including the one studied here.
} 
as $45^{\prime}$ off-axis. PSPC exposure times range from $1 \mathrm{ks}$ to $95 \mathrm{ks}$ for our galaxies. Again, nuclear counts were extracted within a circular region large enough to include all of the associated flux, and the background was measured in a concentric annulus. As with the HRI, we ensured that PSPC backgrounds were well sampled and free of resolved sources. Net count rates for off-axis PSPC sources were corrected for vignetting. We computed upper limits for the four PSPC sources that were not detected. For each of the 46 galaxies observed, Table 2 lists the instrumentation used along with the associated exposure time, background-subtracted nuclear count rate, and off-axis angle.

\section{Analysis and Results}

The ROSAT data provide information about the luminosities and the spatial and spectral characteristics of our galaxies in the soft X-ray band. These properties are useful for comparing different subclasses of objects and for quantifying the LLAGN contribution to the X-ray background. We begin with an investigation of the spatial profiles of the nuclear X-ray sources. We then present spectral analysis and our determination of the nuclear X-ray fluxes for the entire sample, along with a comparison to previously published results.

\subsection{Spatial Analysis}

Our investigation of the soft X-ray spatial characteristics of the galaxies in the LLAGN sample involves a comparison of their azimuthally averaged radial count distributions (radial profiles) to theoretical point-spread functions (PSFs) for the ROSAT instruments. Since our primary objective

is to study the X-ray emission associated with the galaxy nuclei, we have omitted any discrete extranuclear sources from our analysis. We used the "centroid" procedure in the XIMAGE software to determine the position of the central X-ray source in each object. To confirm that the Xray emission is indeed associated with the nucleus, we compared the centroid with the measured optical position reported by Cotton, Condon, \& Arbizzani (1999), which is accurate to $\sim 2^{\prime \prime}$. The optical/X-ray position offsets $\Delta_{\mathrm{OX}}$ are listed in the last column of Table 2 for both the PSPC and HRI data. Most of the X-ray centroids are within a few arcseconds of the optical nucleus, and all are within $20^{\prime \prime}$. Values of $\Delta_{\text {Ox }}$ obtained with the PSPC, despite the lower angular resolution of that instrument, are comparable to those obtained with the HRI. Given the expected uncertainty in the X-ray source positions (see Roberts \& Warwick 2000), the small optical/X-ray offsets suggest that the central X-ray source in each galaxy is associated with the optical nucleus.

Because of the broadening of the ROSAT PSF with off-axis angle and other limitations imposed by the detectors, we have restricted spatial analysis to sufficiently bright sources ( $>50$ counts net), and in the case of the PSPC, to sources detected within $18^{\prime}$ of the optical axis, where the window support ring is located. A total of 31 different objects (22 observed with HRI and 28 observed with 
PSPC) satisfy these criteria. We used the FTOOLS programs extrpsf and calcrpsf to generate a radial profile for the nuclear sources and a theoretical PSF corresponding to their location on the detector. The accuracy of the radial profile is somewhat sensitive to the adopted position of the X-ray source - off-center positions diminish the peak of the profile and broaden it as well. Therefore, for a few point-like sources, we constructed radial profiles using both the measured centroid and adjacent pixels as the source position. This verified that the centroids yielded by XIMAGE are accurate.

Examples of radial profiles covering the range of characteristics present in the LLAGN sample (point-like, moderately resolved, and very extended) are displayed in Figure 1. The theoretical PSF is plotted with each profile. Because of variations in the shape of the PSF, the quality of the detections, and distances of the sources, it is difficult to provide a uniform measure of source extent for the sample. To approximate how point-like each source is, we have computed the ratio of the maximum number of counts expected for a point source (from the theoretical PSF) to the total counts observed. For the measurement, we scaled the PSF such that it (1) peaks at a surface brightness $1 \sigma$ higher than that of the innermost bin of the radial profile, and (2) has a zero-point consistent with the measured background in the image. The derived ratio is therefore a conservative upper limit on the fraction of the observed counts that can be associated with a point source. The results, for both the PSPC and HRI profiles, are listed in Table 3. As the Table shows, only a handful of the sources can be considered point-like; the central X-ray emission in most of the galaxies appears to have a significant extended component, even at the resolution of the PSPC. This comes as a mild surprise, given that our sample consists of AGNs. When galaxies have been observed with both the PSPC and HRI, the HRI point-source fractions are almost always lower, indicating that the source extents are real.

In principle, we could use the derived point-source fractions - particularly those obtained with the HRI - to improve our estimates of the nuclear X-ray flux of each galaxy. However, because high-quality HRI profiles are available for only some of the sources, it would be impossible to apply corrections to the entire sample in a uniform manner. On the other hand, some of the sources are so extended that we can be certain nuclear components make only minor contributions to their total fluxes. Several of these are among the most X-ray-luminous objects in the sample, and failure to correct for the extended emission would severely distort the mean AGN-related luminosity. Thus, for eight objects in Table 3 with PSPC point-source fractions less than $60 \%$ and high-quality PSPC profiles (i.e., NGC 2841, 4258, 4472, 4486, 4636, 4736, 5194, and 5195), we extracted all counts associated with the central source and adjusted the total nuclear X-ray fluxes derived from spectral fitting (discussed in the next section) by the PSPC point-source fraction.

The point-source fraction limits obtained for the remainder of the galaxies are generally too uncertain for this technique to be used reliably. Therefore, for all other objects, we have adopted a consistent procedure for the determination of nuclear fluxes that is governed by the PSPC source characteristics, since most of the objects were observed with that instrument. Admittedly, because of the modest angular resolution of the PSPC, nuclear fluxes will be overestimated in some cases. 
The radial profiles of a number of the nuclear sources indicate that the majority of the extended PSPC emission lies outside the radius that encloses most of the flux of a point source. Thus, one way to reduce the contamination of the nuclear X-ray flux by an extranuclear component would be to extract a smaller area around the source that contains most of the flux of a point source and excludes a major fraction of a non-nuclear component. For each object detected with the PSPC

(except the eight very extended sources discussed above), we used the theoretical PSFs to determine the radius that would include $95 \%$ of the counts in a point source. We then extracted counts from within that radius, which is approximately $1^{\prime}$ for on-axis observations. (The excluded $5 \%$ of the point-source flux is added back to the measured flux.) We also used a local background, starting $1.5^{\prime}$ from the edge of the source region, in order to subtract off some of the extended component. This procedure does not remove all of the effects of extended emission; but unlike the point-source fractions derived above, which are upper limits whose quality varies considerably from case to case, this procedure works uniformly well for all sources regardless of source distance, off-axis angle, and detection strength.

\subsection{Spectral Analysis and Luminosity Measurements}

Based on the quality of the ROSAT detections, we selected a sample of objects for spectral analysis with XSPEC. We found that a spectrum with at least 300 net PSPC source counts is required for reliable model fitting. In preparation for fitting, spectra were grouped to have a minimum of 25-50 counts per energy bin, and effective-area response files were generated using the FTOOLS task pcarf. In each case, the PSPC response matrix appropriate for the gain setting of the detector was used. Because the data have limited spectral resolution and signal-to-noise ratio $(S / N)$, they can only be fitted with relatively simple models. AGNs often have power-law X-ray spectra, so we first attempted to fit each spectrum with a simple absorbed power-law model. In a number of cases, this provided a satisfactory fit (i.e., a reduced $\chi^{2}$ of 1.3 or less). When power-law fits were unacceptable, we tried a two-component model consisting of a power law plus a Raymond-Smith thermal plasma (Raymond \& Smith 1977) with solar abundances. This type of model was found to be useful for analysis of ASCA spectra of LLAGNs (Ptak et al. 1999; Terashima, Ho, \& Ptak 2000). In the two-component models, the absorption column density of the Raymond-Smith component was fixed to the Galactic value, because we expect this emission to arise from an extranuclear source that is not significantly absorbed. Note that seven of the eight highly extended sources discussed in $\S 4.1$ require a Raymond-Smith component. For the power-law component, the absorption was constrained to be greater than or equal to the Galactic value. For almost all of the strongly detected objects, we achieved a very good fit with one of these two models.

We want to stress that our main purpose for modeling the spectra is to determine accurate fluxes and luminosities of the galaxies in the sample. While it is tempting to try to use the spectral parameters associated with the best-fit models for quantitative comparisons, high- $S / N$ X-ray observations of AGNs with good energy resolution invariably reveal complex spectral features, and 
the possibility of contamination by extranuclear emission in these low-luminosity sources leaves us very suspicious of the correspondence between our best-fit models and reality. We have decided, therefore, not to report the fit parameters. Nonetheless, it should be noted that the spectral parameters from our fits are largely consistent with expected values for both high- and low-luminosity AGNs (Walter \& Fink 1993; Ptak et al. 1999). For power-law components, the photon index ranges from 1.1 to 4.3 with a typical value of 2.5 , and for Raymond-Smith components, $k T$ ranges from $0.42 \mathrm{keV}$ to $1.1 \mathrm{keV}$ with a typical value of $0.6 \mathrm{keV}$. For each of the galaxies whose spectrum we modeled, we list in Table 4 the Galactic neutral hydrogen column density, the type of model used, the reduced $\chi^{2}$ for the fit, the observed flux, and the luminosity (corrected for Galactic absorption only).

For the weaker PSPC-observed objects (those with fewer than 300 source counts) and those observed only with the HRI or the Einstein IPC, we must adopt a spectral model in order to convert their detected count rates to fluxes. Under the assumption that these sources are similar to the 20 galaxies in our sample whose spectra we have modeled, we can use the spectral fitting results obtained above to derive an average model suitable for this purpose. Unfortunately, as Table 4 indicates, both one- and two-component models were needed to obtain good fits, which complicates the procedure. However, we have identified a simple one-component (power-law) model that allows us to estimate source fluxes reliably regardless of the intrinsic form of the spectrum. The power-law components used in the models for the objects listed in Table 4 have a typical photon index of 2.5 and a column density slightly higher than the Galactic column. Therefore, we have selected as a typical model a power law with a photon index $\Gamma=2.5$ and column density $N_{\mathrm{H}}=$ Galactic + $2 \times 10^{20} \mathrm{~cm}^{-2}$. To test the accuracy of the fluxes predicted by our "standard" model, we applied it to the 20 well-detected objects for which spectral fitting was possible. We used the PIMMS software to derive, from the standard model and the observed count rates, fluxes corrected for Galactic absorption within the region containing $95 \%$ of the PSF counts. Figure 2 shows a comparison of the flux implied by the standard model and that derived from the spectral fitting. The straight line indicates $F($ model $)=F$ (fitted). As Figure 2 illustrates, the predicted and observed fluxes agree very well over the entire range.

Before we apply the standard model to the HRI-only and weak PSPC sources, we would like to be certain that the spectra of the stronger sources are indeed representative of the spectra of all the objects in the sample. While we cannot produce high-quality spectra for objects with few PSPC counts, we can characterize their spectra using a "hardness ratio." The hardness ratio is defined as $(H-S) /(H+S)$, where $H$ is the number of counts in a hard energy band and $S$ is the number of counts in a soft band. We chose $0.7 \mathrm{keV}$ as the cutoff energy between the hard and soft bands and computed hardness ratios for all objects detected with the PSPC. Hardness ratios span a fairly wide range for both the well-detected objects (those with more 300 PSPC counts) and the weaker sources (those with fewer than 300 counts). To determine whether the hardness ratio distributions of these two groups are similar (i.e., whether they could arise from the same parent distribution), we have compared them using a Kolmogorov-Smirnov test. The cumulative 
distribution functions for the hardness ratios of the two groups of sources are shown in Figure 3. The maximum value of the absolute difference between the functions is $D=0.25$. The probability that $D$ would be greater than 0.25 if the data sets were drawn from the same distribution is 0.67 . Thus, the two distributions of hardness ratios are consistent with a single parent distribution. This indicates that the spectral properties of the weak sources and those of the strong ones do not differ significantly, and furthermore, that application of the standard model to estimate fluxes for the weak-PSPC and HRI-only ROSAT sources is appropriate. These fluxes are also listed in Table 4. Likewise, for the two objects observed by Einstein, the $0.2-3.5 \mathrm{keV}$ IPC count rates from Fabbiano et al. (1992) were converted to $0.1-2.4 \mathrm{keV}$ fluxes using the same model.

Uncertainties in the absorption-corrected fluxes depend upon the method by which they were obtained. For the fluxes measured directly from the spectra, the flux uncertainty depends mainly upon the quality of the fit to the spectrum. Typical uncertainties for these fluxes are $\pm 10 \%$ or less. Fluxes of the remaining objects were based on both the count rate and use of the standard spectral model, each of which has associated uncertainties. The uncertainty in the observed counts $N$ (i.e., $N^{-\frac{1}{2}}$ ) ranges from about $6 \%$ to about $14 \%$, depending on the object. For the uncertainty associated with the application of the standard model, recall that we tested the model by applying it to all the bright objects with high-quality spectra (Fig. 2). Assuming the distribution of $F$ (model)/F(fitted) for these sources is Gaussian, we measure a root-mean-square (rms) deviation of 0.11 from the mean ratio. For a given source, then, the $68 \%$ confidence interval on the flux predicted by the standard model is approximately $\pm 11 \%$. The uncertainties associated with the count rate and use of the standard model are independent, so we may add them in quadrature to estimate the overall uncertainty in the flux and luminosity. In the worst cases, the flux uncertainty for these sources is about $20 \%$.

\section{3. $\quad L_{\mathrm{X}}$ vs. $L_{\mathrm{H} \alpha}$ : Predicting X-ray Fluxes of Unobserved Galaxies}

A correlation has been established between the soft X-ray luminosities and $\mathrm{H} \alpha$ luminosities of AGNs (Elvis, Soltan, \& Keel 1984; Koratkar et al. 1995). In principle, a similar relationship amongst the LLAGNs in our sample would permit us to estimate X-ray fluxes for those members of the sample that have not been observed with ROSAT or Einstein. This is desirable for calculation of the total X-ray volume emissivity represented by this sample, which factors into our estimates of the LLAGN contribution to the soft X-ray background ( $§ 5.3)$.

In Figure 4, we have plotted the X-ray luminosities for the objects listed in Table 4 against their extinction-corrected $\mathrm{H} \alpha$ luminosities from Table 1. The plot includes objects with upper limits on $L_{\mathrm{X}}$ and $L_{\mathrm{H} \alpha}$. The typical $10 \%$ uncertainty in $L_{\mathrm{X}}$ for the objects with fitted spectra is approximately equivalent to the size of the symbols plotted, and a typical uncertainty of about

$16 \%$ for the weaker PSPC sources is shown in the upper left corner of Figure 4. For most objects, the uncertainty in $L_{\mathrm{H} \alpha}$ is between $10 \%$ and $30 \%$ - exceptions are described in the Notes to Table 1. An uncertainty of $30 \%$ in $L_{\mathrm{H} \alpha}$ is also indicated in the upper left corner of Figure 4 . A comparison 
of the typical error bars to the distribution of the points in the Figure suggests that the scatter in the plot is real, and not solely due to errors in our fluxes. Our results are in good agreement with those of Roberts \& Warwick (2000), who investigated the $L_{\mathrm{X}}-L_{\mathrm{H} \alpha}$ correlation in their sample of galaxies (consisting of a wide variety of classifications) observed with the ROSAT HRI.

Despite the scatter, we find a correlation between the soft X-ray and $\mathrm{H} \alpha$ luminosities of the LLAGNs in our sample. The generalized Kendall's $\tau$ correlation test (Isobe, Feigelson, \& Nelson 1986), which properly accounts for the presence of censored data, yields $\tau=0.56$ and $Z=3.01$, corresponding to a probability of 0.003 that the two luminosities are uncorrelated. This result is not a spurious distance effect. To investigate the consequence of the mutual dependence of $L_{\mathrm{X}}$ and $L_{\mathrm{H} \alpha}$ on distance, we applied the partial Kendall's $\tau$ test (Akritas \& Siebert 1996) using distance as the third variable. The partial Kendall's $\tau$ coefficient is 0.29 and the square root of the variance is 0.087 (see Akritas \& Siebert), which imply that the null hypothesis of no correlation between $L_{\mathrm{X}}$ and $L_{\mathrm{H} \alpha}$ can be rejected at a significance level of 0.001. In terms of a Gaussian probability distribution, this is equivalent to $\sim 3 \sigma$. Thus, we feel that the correspondence between the two luminosities is significant enough to permit use of the relationship for predicting X-ray luminosities of the 14 galaxies in our sample lacking ROSAT data.

The individual $L_{\mathrm{X}} / L_{\mathrm{H} \alpha}$ ratios for the sources with high-quality luminosity measurements range from about 1 to 100. This is similar to the range found by Koratkar et al. at higher luminosities $\left(10^{40}<L_{\mathrm{X}}<10^{46} \mathrm{ergs} \mathrm{s}^{-1}\right)$. The median $L_{\mathrm{X}} / L_{\mathrm{H} \alpha}$ ratio for our sample of LLAGNs is 7 , which is somewhat close to the average ratio of 14 found for the five least luminous sources $\left(\sim 10^{40}-10^{41}\right.$ $\operatorname{ergs~}^{-1}$ ) in the Koratkar et al. (1995) study. We use this median ratio, represented by the line in Figure 4, to estimate X-ray luminosities for the 14 galaxies in Table 1 that lack soft X-ray data. The distributions of $L_{\mathrm{X}} / L_{\mathrm{H} \alpha}$ for the Seyferts and LINERs are virtually identical (we return to this point below), so we may apply the conversion independent of the source classification.

\subsection{Previous Flux Measurements}

Several of the objects in our sample are well-known galaxies for which ROSAT observations have already been published. We have employed a uniform procedure for the determination of fluxes and luminosities of the galaxies, which affords consistent results for our large sample. But by its nature, such an approach can be insensitive to particular details associated with specific objects. Thus, it is useful to compare the fluxes and luminosities we have derived with those obtained previously as a result of detailed analyses of the PSPC data.

The fluxes that other investigators have reported are for the $0.1-2.4 \mathrm{keV}$ band and are corrected for Galactic absorption only, except as otherwise noted. Note that ROSAT data for other well-

known galaxies (such as NGC 1068 and NGC 4374) have been published, but fluxes and spectral modeling have not been reported.

$N G C$ 4051. Komossa \& Fink (1997) used a warm absorber model to derive an X-ray flux 
of $4.0 \times 10^{-11}$ ergs $\mathrm{cm}^{-2} \mathrm{~s}^{-1}$. While this is higher than the flux we measured, they also used a different PSPC observation, in which the count rate of this highly variable object was greater than the count rate for the observation we selected.

$N G C$ 4258. A number of sources in the vicinity of this object were investigated by Vogler \& Pietsch (1999). In an attempt to isolate nuclear counts, they extract counts from a very small central region (of radius 24", corresponding to the FWHM of the on-axis PSPC PSF at $1.0 \mathrm{keV}$ ), and subtract off a local background. Their best-fit model for the spectrum of the nuclear region consists of a thermal bremsstrahlung with $k T=0.63 \mathrm{keV}$. Their resulting flux is very close to the nuclear flux that we estimated by lowering the total flux by the fraction included in a theoretical point source (see $\S 4.1)$.

$N G C$ 4278. Koratkar et al. (1995) fitted the spectrum with an absorbed power-law model. They report an unabsorbed $0.2-2.2 \mathrm{keV}$ flux of $2.56 \times 10^{-12} \mathrm{ergs}^{-2} \mathrm{~s}^{-1}$. Note that this is corrected not only for Galactic absorption, but also for the total best-fit column density, which exceeds the Galactic value. If we adopt the same waveband and correct for the same column density, we obtain about half their flux. Their measured count rate is not listed, but since we used spectral parameters very similar to theirs, they presumably extracted counts from a bigger region than we did.

NGC 4388. Antonelli, Matt, \& Piro (1997) fitted a simple absorbed power law to obtain a flux of $6 \times 10^{-13} \mathrm{ergs} \mathrm{cm}^{-2} \mathrm{~s}^{-1}$, which is consistent with our value within the expected errors. However, they propose that a Raymond-Smith model with low metal abundance provides a better description of the spectrum.

$N G C$ 4395. Using an absorbed power-law model, Moran et al. (1999) found a mean 0.2-2.0 $\mathrm{keV}$ flux of about twice our value, even though they measured approximately the same count rate from the same data. Their best-fit model indicated a much flatter power law $(\Gamma=0.9)$ than that which we have adopted here. Thus, in this case, different assumptions about the spectrum have led to different fluxes.

$N G C$ 4450. Komossa, Bohringer, \& Huchra (1999) found, as we did, that a single power law with $N_{\mathrm{H}}$ close to the Galactic value provides an excellent fit to the soft X-ray spectrum. From this model, they derived a flux consistent with our own.

$N G C$ 4486. Prieto (1996) attempted to determine the nuclear flux by subtracting off a local background and fitting the "residual" nuclear spectrum with a power law. The resulting upper limit is about twice our value. We have used a different strategy for isolating the nuclear flux, as described in $\S 4.1$, and expect that our value represents a tighter constraint on the output of the nucleus.

$N G C$ 4565. Optically thin thermal plasma and power-law models were tested by Vogler, Pietsch, \& Kahabka (1996) on various sources associated with this object. For the central source, both models lead to fluxes consistent with ours. 
$N G C$ 4636. Trinchieri et al. (1994) fitted the spectrum separately in concentric annuli around the nucleus. Using the $10^{\prime}-15^{\prime}$ annulus as a local background, they obtain a $0.1-2.0 \mathrm{keV}$ flux of $9.38 \times 10^{-12} \mathrm{ergs} \mathrm{cm}^{-2} \mathrm{~s}^{-1}$ from a central region of radius $8^{\prime}$. This is comparable to the flux that we derived for the entire extended source, but we substantially reduced this flux by including only the fraction contained within a point source.

$N G C$ 4639. Koratkar et al. (1995) fitted the spectrum with an absorbed power law of photon index 2.27. The resulting unabsorbed $0.2-2.2 \mathrm{keV}$ flux is somewhat higher than ours, but it has been corrected for a best-fit column density that exceeds the Galactic $N_{\mathrm{H}}$. If we use the same column density in our model, we obtain a similar flux.

$N G C$ 4736. A two-component model similar to ours, consisting of a power law and a RaymondSmith plasma, was favored by Cui, Feldkhun, \& Braun (1997). Significant deviation at the lowenergy end of the spectrum necessitated the addition of a Gaussian line feature centered at 0.22 $\mathrm{keV}$. Altering elemental abundances and experimenting with more complicated models failed to improve the low-energy fit, so they suggested that the Gaussian component may be a calibration artifact. They derived an observed $0.1-2.0 \mathrm{keV}$ flux of $1.78 \times 10^{-12} \mathrm{ergs} \mathrm{cm}^{-2} \mathrm{~s}^{-1}$, which is very close to our observed flux before we attempted to minimize the included flux due to extended emission. Again, we have attempted to estimate only the flux arising from a point source, so we expect that our value is closer to the flux produced by the nucleus itself.

As a whole, while some differences exist between our fluxes and those measured previously, most appear to be due to variability, different extractions of counts, or our attempts to eliminate extended emission, rather than substantially different spectral models. Thus, we have confidence in the statistical reliability of our relatively uniform approach.

\section{Discussion}

\subsection{Comparing LINERs and Seyfert Galaxies}

Comparison of the soft X-ray properties of Seyfert galaxies and LINERs may help to determine whether they share a similar type of power source. The ROSAT data we have assembled are particularly suited for such a comparison, since our sample of LLAGNs is volume-limited and complete. Therefore, we do not expect selection effects to bias our conclusions.

Table 5 lists the median values of X-ray luminosity and $L_{\mathrm{X}} / L_{\mathrm{H} \alpha}$ for the various subtypes of galaxies in the sample. We also list the interquartile ranges, which contain the middle $50 \%$ of the values. Because these values include limits, we used the Kaplan-Meier product-limit estimator (Feigelson \& Nelson 1985) to compute the medians and quartiles. In Figure 5, we compare the distributions of $L_{\mathrm{X}}$ and $L_{\mathrm{X}} / L_{\mathrm{H} \alpha}$ for Seyfert galaxies and LINERs; upper limits are indicated by arrows. Examination of Figure 5 and Table 5 reveals that the typical X-ray luminosity of Seyferts is very close to that of LINERs, and that the distributions of $L_{\mathrm{X}} / L_{\mathrm{H} \alpha}$ for Seyferts and LINERs 
also appear very similar. We have applied statistical tests for censored univariate data in order to quantify the apparent similarity. The Gehan and logrank tests (Feigelson \& Nelson 1985) indicate, respectively, $60 \%$ and $98 \%$ probabilities that the $L_{\mathrm{X}}$ samples arise from the same parent population, and $19 \%$ and $22 \%$ probabilities that the $L_{\mathrm{X}} / L_{\mathrm{H} \alpha}$ distributions are the same. Because two distributions are not considered statistically different unless the null hypothesis can be rejected at less than $5 \%$, we conclude that $L_{\mathrm{X}}$ and $L_{\mathrm{X}} / L_{\mathrm{H} \alpha}$ are indeed statistically similar for our Seyferts and LINERs.

Although we have not presented the details of the spectral modeling used to measure the fluxes of the well-detected LLAGNs, we found no significant differences between the soft X-ray spectral characteristics of the Seyferts and LINERs. For example, Seyfert and LINER spectra were equally likely to require a Raymond-Smith component in addition to a power law. The luminosity and spectral similarities may therefore be an indication that, in general, Seyferts and LINER nuclei are powered by the same physical mechanism. The existence of a correlation between the X-ray and $\mathrm{H} \alpha$ fluxes of the galaxies in our sample and the absence of any segregation of the Seyferts and LINERs in Figure 4 provides additional support for this conclusion.

We note, however, that many of our objects exhibit significant extended X-ray emission, which complicates the determination of the X-ray flux associated with the nucleus. As discussed in $\S 4$, we have attempted to minimize the contribution of extended emission in our flux measurements. Nevertheless, the possibility remains that extranuclear components are responsible for non-negligible amounts of the measured flux in many sources - even those that are approximately point-like - due to the modest angular resolution of the PSPC. The lower point-source fractions obtained from HRI observations of the same sources (see $\S 4.1$ and Table 3 ) suggest this might be the case. Thus, the similarities of the mean soft X-ray luminosities and $L_{\mathrm{X}} / L_{\mathrm{H} \alpha}$ ratios for Seyfert galaxies and LINERs may be due to the presence of significant amounts of circumnuclear extended emission in both types of objects. If so, it would be premature to conclude that their power sources are similar.

As a preliminary test of this possibility, we have attempted to measure objectively the role of extended emission in the objects for which we constructed HRI radial profiles. At the distance limit of our sample $(18 \mathrm{Mpc})$, more than $90 \%$ of the energy in the HRI PSF is contained within a radius of $\sim 0.2^{\prime}$, which corresponds to a physical radius of $\sim 1 \mathrm{kpc}$. We have therefore defined a parameter $\xi=$ (source counts detected beyond $1 \mathrm{kpc}) /$ (total source counts) $-f$, where $f$ is the fraction of the energy in the PSF found at $r>1 \mathrm{kpc}$ (for the most distant sources, $f$ has a maximum value of $\sim 0.07)$. Thus, $\xi$ will have a value of zero for an unresolved source and a value near unity for a very extended source; in general, it represents a lower limit on the fraction of the central X-ray emission produced by non-nuclear components. We have measured $\xi$ for all sources with HRI radial profiles. The distribution of $\xi$ values for Seyferts and LINERs is plotted in Figure 6. The distributions are quite similar, indicating that the low-luminosity Seyferts and LINERs have, in a statistical sense, comparable amounts of extended central X-ray emission. Of course, this is a very tentative conclusion-HRI profiles are available for only a fraction of our sample, and their quality varies considerably from source to source. 
In summary, the soft X-ray properties of our LLAGN sample provide ambiguous evidence regarding the existence of a common excitation mechanism in Seyfert galaxies and LINERs. On

one hand, we would not expect Seyferts and LINERs to occupy the same region in Figure 4 if the majority of LINERs are powered by stellar processes rather than accretion onto a massive compact object. On the other hand, the $L_{\mathrm{X}}-L_{\mathrm{H} \alpha}$ correlation we have obtained is not very strong, and we have demonstrated clearly that a significant fraction of the central X-ray emission in LLAGNs (of all types) is extranuclear in origin. A definitive comparison of the X-ray properties of Seyfert galaxies and LINERs will thus require the angular resolution and energy range afforded by Chandra.

\subsection{Comparing Type 1 and Type 2 AGNs}

The unified model for AGNs holds that the principal difference between type 1 and type 2 objects is their orientation with respect to the viewer (e.g., Antonucci 1993). We observe a type 1 AGN when we look directly into the broad-line region, whereas type 2 objects are observed when the broad-line region is obscured by an optically thick torus. If the unified model is universal, we would expect type 1 galaxies to have generally higher soft X-ray luminosities than type 2s. Furthermore, because of the additional absorbing material along the line of sight to type 2 nuclei, $L_{\mathrm{X}} / L_{\mathrm{H} \alpha}$ should be significantly greater in type 1 objects.

The distributions of these quantities are shown in Table 5. While $L_{\mathrm{X}}$ tends to be somewhat higher for type $1 \mathrm{~s}$ than type 2 s (see Table 7), the Gehan and logrank tests give $5 \%$ and $6 \%$ probabilities that the $L_{\mathrm{X}}$ samples are the same, indicating that $L_{\mathrm{X}}$ may tend to be marginally higher for type 1 objects. For the $L_{\mathrm{X}} / L_{\mathrm{H} \alpha}$ distributions, however, the tests indicate $13 \%$ and $11 \%$ probabilities, respectively, that the samples arise from the same parent population, so we are unable to conclude that a substantial difference exists. At face value, then, these data do not strongly support the general applicability of the unified model. If we assume that the majority of the Xray luminosity actually arises from an AGN in most cases, the similarity of the $L_{\mathrm{X}}$ and $L_{\mathrm{X}} / L_{\mathrm{H} \alpha}$ distributions suggests that type 2 objects are not, in fact, significantly more absorbed than type 1 objects. On the other hand, the spectra of type 2 objects are somewhat more likely to require a Raymond-Smith component, and we have found that many of the galaxies in our sample have substantial extended components that may be contaminating their nuclear emission. Since we do not expect the flux produced outside the nucleus to be highly absorbed, such contamination may be masking any intrinsic differences between the soft X-ray properties of type 1 and type 2 nuclei.

\subsection{Contribution of LLAGNs to the Cosmic X-ray Background}

A substantial fraction of the cosmic X-ray background (XRB) is known to arise from QSOs and other highly luminous AGNs. However, the luminosity function, clustering properties, and X-ray spectral characteristics of such objects indicate that they cannot account for all of the observed 
background (Fabian \& Barcons 1992). Contributions from other types of sources, such as non-AGN galaxies and/or obscured LLAGNs, may be required to provide a full description of the observed XRB (Comastri et al. 1995). In this section, we attempt to quantify the XRB contribution of low-luminosity Seyferts and LINERs such as those in our sample.

First, we must compute the local soft X-ray volume emissivity of LLAGNs. Our unbiased sample allows us to estimate this quantity accurately. To avoid the possibility of incompleteness in the "zone of avoidance" we have only included galaxies that are more than $30^{\circ}$ from the Galactic plane (this is 53 of the objects in our sample). In this region, the total $0.1-2.4 \mathrm{keV}$ luminosity of the objects that were observed by ROSAT is $1.70 \times 10^{42} \mathrm{ergs} \mathrm{s}^{-1}$ (uncorrected for absorption). The sources lacking ROSAT data (see $\S 4.3$ ) contribute an estimated additional $1.16 \times 10^{41} \mathrm{ergs} \mathrm{s}^{-1}$, for a total energy output of $1.81 \times 10^{42} \mathrm{ergs} \mathrm{s}^{-1}$ from LINERs and Seyferts. Assuming a powerlaw spectrum with $\Gamma=2.5$ and $N_{\mathrm{H}}=4 \times 10^{20} \mathrm{~cm}^{-2}$, this translates to an absorption-corrected volume emissivity of $2.20 \times 10^{38} \mathrm{ergs} \mathrm{s}^{-1} \mathrm{Mpc}^{-3}$ in the $0.5-2.0 \mathrm{keV}$ band. This result is in close agreement with the value of $2.27 \times 10^{38} \mathrm{ergs} \mathrm{s}^{-1} \mathrm{Mpc}^{-3}$ obtained for low-luminosity X-ray galaxies via optical/X-ray cross-correlation techniques (Almaini et al. 1997).

Assuming that the LLAGN volume emissivity does not evolve with redshift, we have computed the XRB intensity produced by such objects by integrating eq. (18) from Soltan et al. (1996):

$$
I_{\mathrm{X}}=\frac{c}{4 \pi H_{0}} \rho \int_{0}^{z_{\max }} \frac{(1+z)^{1-\alpha}}{(1+z)^{3} \sqrt{1+\Omega_{\mathrm{M}} z}} d z
$$

where $\rho$ is the volume emissivity, $\Omega_{\mathrm{M}}$ is the density parameter, $z$ is the redshift, and $\alpha(=\Gamma-1)$ is the average energy index of their power-law X-ray spectra. We chose $z=5$ as the upper limit of integration; the calculation is not sensitive to this choice, since more than $95 \%$ of the XRB contribution is produced at $z<2$ in the absence of evolution. We assume the total $0.5-2 \mathrm{keV}$ $\mathrm{XRB}$ intensity is $2.61 \times 10^{-8} \mathrm{ergs} \mathrm{cm}^{-2} \mathrm{~s}^{-1} \mathrm{sr}^{-1}$ (Soltan et al. 1996). In an $\Omega_{\mathrm{M}}=1.0, \Omega_{\Lambda}=0.0$ cosmology, we find that nonevolving LLAGNs produce $9 \%$ of the soft XRB. Generalizing the Soltan et al. equation for an $\Omega_{\mathrm{M}}=0.3, \Omega_{\Lambda}=0.7$ cosmology (e.g., Garnavich et al. 1998; Balbi et al. 2000; Hanany et al. 2000), we find their contribution is 11\%. A higher fraction of the soft XRB will be accounted for by LLAGNs if their volume emissivity evolves with redshift. We note, however, that if LLAGNs were to evolve as strongly as high-luminosity AGNs (see Miyaji, Hasinger, \& Schmidt 2000), they would overproduce the soft XRB. This supports the conclusion of Miyaji et al. that there is a strong luminosity dependence on the evolution of the AGN X-ray luminosity function.

To compare our results with recent estimates of the soft XRB contribution from higher luminosity AGNs, we consider the findings from the ROSAT Deep Survey of the "Lockman Hole." Hasinger et al. (1998) resolved $68 \%-81 \%$ of the $1-2 \mathrm{keV}$ X-ray background in this field, depending on the actual level and spectrum of the XRB in that energy range. Subsequent optical follow-up by Schmidt et al. (1998) revealed that 39 of the 50 sources resolved in the survey are luminous AGNs (most having luminosities between $10^{43}$ and $10^{45} \mathrm{ergs} \mathrm{s}^{-1}$ ), indicating that $53 \%-63 \%$ of the $\mathrm{XRB}$ is produced by luminous AGNs in the $1-2 \mathrm{keV}$ band. In comparison, the values of $9 \%-11 \%$ 
we estimate for the LLAGN contribution to the soft XRB, which are lower limits, appear to be reasonable.

\section{Summary}

We have analyzed the soft X-ray properties of a complete, distance-limited sample of 60 galaxies classified by Ho et al. (1997a) as Seyfert galaxies or LINERs. We find that the central X-ray sources in the majority of the galaxies exhibit significant amounts of extended emission, in addition to the emission from the active nucleus. We have attempted to isolate the nuclear emission as much as possible, guided by spatial information contained in the X-ray images. The spectra of objects with at least 300 net PSPC counts are well fitted with either simple absorbed power-law models, or with two-component models consisting of a power law and a thermal plasma. For these objects, fluxes and luminosities were derived from the spectral modeling; a standard spectral model based on their typical fit parameters was used to determine the fluxes of the weaker sources. We have investigated the relationship between $L_{\mathrm{X}}$ and $L_{\mathrm{H} \alpha}$ for the 46 objects with soft X-ray data, finding a weak correlation between these quantities that is roughly consistent with the correlation found in higher-luminosity AGNs. We have used the median $L_{\mathrm{X}} / L_{\mathrm{H} \alpha}$ ratio of 7 for our sample to predict the X-ray luminosities of the 14 objects lacking soft X-ray fluxes.

We find that low-luminosity Seyferts and LINERs have similar soft X-ray properties. In terms of their X-ray luminosity, $L_{\mathrm{X}} / L_{\mathrm{H} \alpha}$ distribution, and spectral properties, no differences are apparent, suggesting that the Seyferts and the majority of LINERs may be powered by a common mechanism. It is possible, however, that a general presence of significant extended emission in both types of objects is responsible for the apparent similarities. A comparison of the X-ray properties of the type 1 and type 2 AGNs in our sample did not reveal the differences expected if the unified AGN model is universal, although once again, contamination of the nuclear X-ray fluxes by extended emission components could be responsible.

Finally, we have estimated the fraction of the soft X-ray background contributed by lowluminosity Seyferts and LINERs, based on the local $0.5-2.0 \mathrm{keV}$ volume emissivity of $2.2 \times 10^{38}$ ergs s${ }^{-1} \mathrm{Mpc}^{-3}$ derived for our sample of LLAGNs. With no evolution, our results suggest that LLAGNs produce $9 \%-11 \%$ of the XRB in this energy range.

This research has made use of data obtained from the High Energy Astrophysics Science Archive Research Center (HEASARC), provided by NASA's Goddard Space Flight Center. The

work of ECM is supported by NASA through Chandra Fellowship PF8-10004 awarded by the Chandra X-ray Observatory Center, which is operated by the Smithsonian Astrophysical Observatory for NASA under contract NAS8-39073. We also acknowledge the support of NASA through grants NAG 5-3556 and STScI GO-8607. Finally, we would like to thank the anonymous referee for providing many valuable suggestions. 


\section{REFERENCES}

Akritas, M. G., \& Siebert, J. 1996, MNRAS, 278, 919

Almaini, O., Shanks, T., Griffiths, R. E., Boyle, B. J., Roche, N., Georgantopolous, I., \& Stewart, G. C. 1997, MNRAS, 291, 372

Antonelli, L. A., Matt, G., \& Piro, L. 1997, A\&A, 317, 686

Antonucci, R. 1993, ARA\&A, 31, 473

Balbi, A., et al. 2000, ApJ, 545, L1

Barth, A. J., \& Shields, J. C. 2000, PASP, 112, 753

Boyle, B., Shanks, T., Georgantopoulos, I., Stewart, G., \& Griffiths, R. 1994, MNRAS, 271, 639

Comastri, A., Setti, G., Zamorani, G., \& Hasinger, G. 1995, A\&A, 296, 1

Cotton, W. D., Condon, J. J., \& Arbizzani, E. 1999, ApJS, 125, 409

Cui, W., Feldkhun, D., \& Braun, R. 1997, ApJ, 477, 693

Dickey, J. M., \& Lockman, F. J. 1990, ARA\&A, 28, 215

Dopita, M. A., \& Sutherland, R. S. 1995, ApJ, 455, 468

Elvis, M., Soltan, A., \& Keel, W. 1984, ApJ, 283, 479

Elvis, M., \& van Speybroeck, L. 1982, ApJ, 257, L51

Fabbiano, G., Kim, D.-W., \& Trinchieri, G. 1992, ApJS, 80, 531

Fabian, A. C., \& Barcons, X. 1992, ARA\&A, 30, 429

Feigelson, E. D., \& Nelson, P. I. 1985, ApJ, 293, 192

Ferland, G. J., \& Netzer, H. 1983, ApJ, 264, 105

Filippenko, A. V. 1996, in The Physics of LINERs in View of Recent Observations, ed. M. Eracleous et al. (San Francisco: ASP), 17

Filippenko, A. V., \& Sargent, W. L. W. 1985, ApJS, 57, 503

Filippenko, A. V., \& Terlevich, R. 1992, ApJ, 397, L79

Garnavich, P., et al. 1998, ApJ, 509, 74

Georgantopolous, I., Basilakos, S., \& Plionis, M. 1999, MNRAS, 305, L31 
Halpern, J. P., \& Steiner, J. E. 1983, ApJ, 269, L37

Hanany, S., et al. 2000, ApJ, 545, L5

Hasinger, G., Burg, R., Giacconi, R., Schmidt, M., Trümper, J., \& Zamorani, G. 1998, A\&A, 329,482

Heckman, T. M. 1980, A\&A, 87, 142

Heckman, T. M., Balick, B., \& Crane, P. C. 1980, A\&A, 40, 295

Ho, L. C., Filippenko, A. V., \& Sargent, W. L. W. 1993, ApJ, 417, 63

Ho, L. C., Filippenko, A. V., \& Sargent, W. L. W. 1995, ApJS, 98, 477

Ho, L. C., Filippenko, A. V., \& Sargent, W. L. W. 1997a, ApJS, 112, 315

Ho, L. C., Filippenko, A. V., \& Sargent, W. L. W. 1997b, ApJ, 487, 568

Ho, L. C., Filippenko, A. V., Sargent, W. L. W., \& Peng, C. Y. 1997c, ApJS, 112, 391

Ho, L. C., \& Peng, C. Y. 2001, ApJ, in press

Ho, L. C., \& Ulvestad, J. S. 2001, ApJS, 133, 77

Isobe, T., Feigelson, E. D., \& Nelson, P. I. 1986, ApJ, 306, 490

Keel, W. C. 1983, ApJ, 269, 466

Komossa, S., Bohringer, H., \& Huchra, J. P. 1999, A\&A, 349, 88

Komossa, S., \& Fink, H. 1997, A\&A, 322, 719

Koratkar, A. P., Deustua, S., Heckman, T. M., Filippenko, A. V., Ho, L. C., \& Rao, M. 1995, ApJ, 440, 132

Moran, E. C., Filippenko, A. V., Ho, L. C., Shields, J. C., Belloni, T., Comastri, A., Snowden, S. L., \& Sramek, R. A. 1999, PASP, 111, 801

Mulchaey, J. S., Colbert, E., Wilson, A. S., Mushotzky, R. F., \& Weaver, K. A. 1993, ApJ, 414, 144

Prieto, M. A. 1996, MNRAS, 282, 421

Ptak, A., Serlemitsos, P. J., Yaqoob, T., \& Mushotzky, R. 1999, ApJS, 120, 179

Raymond, J. C., \& Smith, B. W. 1977, ApJS, 35, 419

Roberts, T. P., \& Warwick, R. 2000, MNRAS, 315, 98 
Roche, N., Griffiths, R. E., Della Ceca, R., Shanks, T., Boyle, B. J., Georgantopolous, I., \& Stewart, G. C. 1996, MNRAS, 282, 820

Schmidt, M., et al. 1998, A\&A, 329, 495

Shields, J. C. 1992, ApJ, 399, L27

Shuder, J. M. 1981, ApJ, 244, 12

Soltan, A. M., Hasinger, G., Egger, R., Snowden, S., \& Trümper, J. 1996, A\&A, 305, 17

Stauffer, J. R. 1982, ApJ, 262, 66

Terashima, Y., Ho, L. C., \& Ptak, A. F. 2000, ApJ, 539, 161

Trinchieri, G., Kim, D.-W., Fabbiano, G., \& Canizares, C. R. C. 1994, ApJ, 428, 555

Vogler, A., \& Pietsch, W. 1999, A\&A, 352, 64

Vogler, A., Pietsch, W., \& Kahabka, P. 1996, A\&A, 305, 74

Walter, R., \& Fink, H. H. 1993, A\&A, 274, 105

Yee, H. K. C. 1980, ApJ, 241, 894 
Table 1. The Distance-Limited LLAGN Sample

\begin{tabular}{|c|c|c|c|}
\hline Galaxy & Class $^{\mathrm{a}}$ & $D(\mathrm{Mpc})$ & $\log L_{\mathrm{H} \alpha}^{b}$ \\
\hline $\mathrm{IC} 239^{\mathrm{c}}$ & L2:: & 16.8 & $36.89 \mathrm{x}$ \\
\hline NGC 185 & $\mathrm{~S} 2$ & 0.7 & 34.90 \\
\hline NGC 404 & $\mathrm{~L} 2$ & 2.4 & 37.76 \\
\hline $\mathrm{NGC} 428^{\mathrm{c}}$ & L2: & 14.9 & $36.98 \mathrm{n}$ \\
\hline NGC 1052 & L1.9 & 17.8 & $39.80 \mathrm{x}$ \\
\hline NGC 1058 & $\mathrm{~S} 2$ & 9.1 & 38.16 \\
\hline NGC 1068 & $\mathrm{~S} 1.9$ & 14.4 & $41.65 \mathrm{x}$ \\
\hline NGC 2681 & L1.9 & 13.3 & $39.27 \mathrm{n}$ \\
\hline NGC $2683^{\mathrm{c}}$ & L2 & 5.7 & 37.48 \\
\hline NGC $2685^{\mathrm{c}}$ & $\mathrm{S} 2:$ & 16.2 & 39.21 \\
\hline NGC $2787^{\mathrm{c}}$ & L1.9 & 13.0 & 38.95 \\
\hline NGC 2841 & L2 & 12.0 & $38.80 \mathrm{x}$ \\
\hline NGC 3031 & $\mathrm{~S} 1.5$ & 1.4 & 38.53 \\
\hline NGC $3368^{\mathrm{c}}$ & $\mathrm{L} 2$ & 8.1 & $38.91 x$ \\
\hline NGC 3379 & L2:: & 8.1 & $37.94 \mathrm{c}$ \\
\hline NGC 3486 & $\mathrm{~S} 2$ & 7.4 & 37.85 \\
\hline NGC 3623 & L2: & 7.3 & 37.77 \\
\hline NGC $3718^{c}$ & L1.9 & 17.0 & $39.29 \mathrm{n}$ \\
\hline NGC 3982 & $\mathrm{~S} 1.9$ & 17.0 & 39.82 \\
\hline NGC 4051 & $\mathrm{~S} 1.2$ & 17.0 & $40.47 \mathrm{x}$ \\
\hline NGC $4111^{c}$ & L2 & 17.0 & 39.84 \\
\hline NGC 4138 & $\mathrm{~S} 1.9$ & 17.0 & 38.99 \\
\hline NGC $4143^{\mathrm{c}}$ & L1.9 & 17.0 & 39.27 \\
\hline NGC 4168 & S1.9: & 16.8 & 38.40 \\
\hline NGC 4203 & L1.9 & 9.7 & 38.79 \\
\hline NGC 4258 & S1.9 & 6.8 & 39.08 \\
\hline NGC 4278 & L1.9 & 9.7 & 39.38 \\
\hline NGC 4293 & L2 & 17.0 & 39.68 \\
\hline NGC 4314 & $\mathrm{~L} 2$ & 9.7 & 38.56 \\
\hline
\end{tabular}


Table 1-Continued

\begin{tabular}{|c|c|c|c|}
\hline Galaxy & Class $^{\mathrm{a}}$ & $D(\mathrm{Mpc})$ & $\log L_{\mathrm{H} \alpha}^{\mathrm{b}}$ \\
\hline NGC 4346 & L2:: & 17.0 & $37.53 \mathrm{c}$ \\
\hline NGC 4374 & $\mathrm{~L} 2$ & 16.8 & 39.31 \\
\hline NGC 4388 & S1.9 & 16.8 & $40.28 \mathrm{x}$ \\
\hline NGC 4394 & L2 & 16.8 & 38.68 \\
\hline NGC 4395 & S1.8 & 3.6 & 38.67 \\
\hline NGC 4438 & L1.9 & 16.8 & 40.11 \\
\hline NGC 4450 & L1.9 & 16.8 & $38.79 \mathrm{x}$ \\
\hline $\mathrm{NGC} 4457^{\mathrm{C}}$ & $\mathrm{L} 2$ & 17.4 & 39.77 \\
\hline NGC 4472 & $\mathrm{~S} 2::$ & 16.8 & $37.59 \mathrm{c}$ \\
\hline NGC 4477 & $\mathrm{~S} 2$ & 16.8 & 39.06 \\
\hline NGC 4486 & L2 & 16.8 & 39.77 \\
\hline NGC 4494 & L2:: & 9.7 & $37.54 \mathrm{u}$ \\
\hline NGC 4501 & $\mathrm{~S} 2$ & 16.8 & 39.06 \\
\hline NGC 4548 & $\mathrm{~L} 2$ & 16.8 & 38.89 \\
\hline NGC 4550 & $\mathrm{~L} 2$ & 16.8 & $38.51 \mathrm{x}$ \\
\hline NGC 4565 & S1.9 & 9.7 & 38.70 \\
\hline NGC 4579 & S1.9 & 16.8 & 39.72 \\
\hline NGC $4596^{\mathrm{c}}$ & L2:: & 16.8 & $37.95 \mathrm{~b}$ \\
\hline NGC 4636 & L1.9 & 17.0 & 38.63 \\
\hline NGC 4639 & $\mathrm{~S} 1.0$ & 16.8 & 39.77 \\
\hline NGC 4651 & $\mathrm{~L} 2$ & 16.8 & 38.24 \\
\hline NGC 4698 & $\mathrm{~S} 2$ & 16.8 & 38.74 \\
\hline NGC 4725 & $\mathrm{~S} 2:$ & 12.4 & 38.22 \\
\hline NGC 4736 & L2 & 4.3 & $37.81 \mathrm{x}$ \\
\hline NGC 4762 & L2: & 16.8 & $37.49 \mathrm{~b}$ \\
\hline NGC 4772 & L1.9 & 16.3 & $38.95 n$ \\
\hline NGC $4866^{\mathrm{c}}$ & $\mathrm{L} 2$ & 16.0 & 38.64 \\
\hline NGC 5194 & $\mathrm{~S} 2$ & 7.7 & $39.80 \mathrm{x}$ \\
\hline NGC 5195 & L2: & 9.3 & $38.67 x$ \\
\hline NGC $7217^{\mathrm{c}}$ & $\mathrm{L} 2$ & 16.0 & 39.67 \\
\hline
\end{tabular}


Table 1 - Continued

\begin{tabular}{rlcl}
\hline \hline Galaxy & Class $^{\mathrm{a}}$ & $D(\mathrm{Mpc})$ & $\log L_{\mathrm{H} \alpha}{ }^{\mathrm{b}}$ \\
\hline & & & \\
NGC $7814^{\mathrm{c}}$ & $\mathrm{L} 2::$ & 15.1 & $36.83 \mathrm{~b}$ \\
\hline
\end{tabular}

Note. - Data from Ho et al. (1997a).

${ }^{a} \mathrm{~L}=$ LINER, $\mathrm{S}=$ Seyfert; type 2 objects have no detectable broad lines, whereas type 1.9 objects exhibit weak broad $\mathrm{H} \alpha$ emission; luminosity includes broad and narrow components where both exist. Uncertain classifications are followed by a colon; double colons mean that the classification is highly uncertain.

b "x" denotes data from one of the following sources: Stauffer (1982), Keel (1983), or Heckman, Balick, \& Crane (1980). "u" indicates a $3 \sigma$ upper limit, and "b" and "c" are quality ratings corresponding to probable uncertainties of $\pm 30 \%-50 \%$ and $\pm 100 \%$, respectively. "n" indicates data obtained under nonphotometric conditions, which lead to typical uncertainties of $\pm 100 \%$.

${ }^{\mathrm{c}}$ Soft X-ray data are not available. 
Table 2. Soft X-ray Observations

\begin{tabular}{|c|c|c|c|c|c|}
\hline Galaxy & Instrument & Count rate ${ }^{\mathrm{a}}$ & Exp. (s) & Off-axis angle $\left({ }^{\prime}\right)$ & $\Delta_{\mathrm{OX}}\left({ }^{\prime \prime}\right)$ \\
\hline NGC 185 & HRI & $<1.53 \mathrm{E}-3$ & 21070 & 0.9 & $\cdots$ \\
\hline NGC 404 & HRI & $(1.93 \pm 0.28) \mathrm{E}-3$ & 23874 & 0.2 & 4 \\
\hline \multirow[t]{2}{*}{ NGC 1052} & HRI & $(8.64 \pm 0.63) \mathrm{E}-3$ & 22105 & 4.4 & 4 \\
\hline & PSPC & $(3.60 \pm 0.16) \mathrm{E}-2$ & 13975 & 0.4 & 7 \\
\hline NGC 1058 & HRI & $<9.81 \mathrm{E}-4$ & 60796 & 1.3 & $\cdots$ \\
\hline \multirow[t]{2}{*}{ NGC 1068} & HRI & $(5.80 \pm 0.02) \mathrm{E}-1$ & 114768 & 0.2 & 2 \\
\hline & PSPC & $1.85 \pm 0.02$ & 5471 & 0.2 & 6 \\
\hline NGC 2681 & PSPC & $<8.78 \mathrm{E}-3$ & 4648 & 35.3 & $\cdots$ \\
\hline NGC 2841 & PSPC & $(4.71 \pm 0.19) \mathrm{E}-2$ & 13428 & 0.2 & 2 \\
\hline \multirow[t]{2}{*}{ NGC 3031} & HRI & $(2.83 \pm 0.02) \mathrm{E}-1$ & 102000 & 0.3 & 7 \\
\hline & PSPC & $(7.98 \pm 0.04) \mathrm{E}-1$ & 49197 & 0.3 & 7 \\
\hline NGC 3379 & HRI & $(3.22 \pm 0.36) \mathrm{E}-3$ & 24560 & 0.4 & 4 \\
\hline NGC 3486 & HRI & $<1.69 \mathrm{E}-3$ & 15841 & 0.8 & $\cdots$ \\
\hline \multirow[t]{2}{*}{ NGC 3623} & HRI & $(3.74 \pm 0.32) \mathrm{E}-3$ & 36916 & 0.2 & 8 \\
\hline & PSPC & $(1.90 \pm 0.10) \mathrm{E}-2$ & 17494 & 0.2 & 7 \\
\hline NGC 3982 & PSPC & $(1.45 \pm 0.15) \mathrm{E}-2$ & 6264 & 0.4 & 3 \\
\hline \multirow[t]{2}{*}{ NGC 4051} & HRI & $1.08 \pm 0.01$ & 10579 & 0.1 & 7 \\
\hline & PSPC & $1.58 \pm 0.01$ & 28727 & 1.2 & 9 \\
\hline NGC 4138 & HRI & $<3.43 \mathrm{E}-3$ & 5798 & 3.2 & $\ldots$ \\
\hline
\end{tabular}


Table 2-Continued

\begin{tabular}{|c|c|c|c|c|c|}
\hline Galaxy & Instrument & Count rate ${ }^{\mathrm{a}}$ & Exp. (s) & Off-axis angle $\left({ }^{\prime}\right)$ & $\Delta_{\mathrm{OX}}\left({ }^{\prime \prime}\right)$ \\
\hline NGC 4168 & PSPC & $(7.97 \pm 0.75) \mathrm{E}-3$ & 14300 & 31.1 & 17 \\
\hline \multirow[t]{2}{*}{ NGC 4203} & HRI & $(5.39 \pm 0.14) \mathrm{E}-2$ & 25448 & 1.0 & 5 \\
\hline & PSPC & $(2.34 \pm 0.03) \mathrm{E}-1$ & 22663 & 1.0 & 5 \\
\hline \multirow[t]{2}{*}{ NGC 4258} & HRI & $(8.74 \pm 0.18) \mathrm{E}-2$ & 27556 & 0.4 & 14 \\
\hline & PSPC & $(2.96 \pm 0.03) \mathrm{E}-1$ & 32864 & 0.3 & 15 \\
\hline \multirow[t]{2}{*}{ NGC 4278} & HRI & $(1.64 \pm 0.13) \mathrm{E}-2$ & 9986 & 0.1 & 4 \\
\hline & PSPC & $(5.45 \pm 0.40) \mathrm{E}-2$ & 3411 & 0.1 & 10 \\
\hline NGC 4293 & PSPC & $(6.11 \pm 0.59) \mathrm{E}-3$ & 17507 & 0.7 & 11 \\
\hline NGC 4314 & PSPC & $(1.20 \pm 0.10) \mathrm{E}-2$ & 10956 & 22.9 & 7 \\
\hline NGC 4346 & PSPC & $<1.83 \mathrm{E}-2$ & 5510 & 42.3 & $\cdots$ \\
\hline \multirow[t]{2}{*}{ NGC 4374} & HRI & $(4.80 \pm 0.13) \mathrm{E}-2$ & 26490 & 0.3 & 3 \\
\hline & PSPC & $(1.38 \pm 0.03) \mathrm{E}-1$ & 22020 & 17.1 & 9 \\
\hline \multirow[t]{2}{*}{ NGC 4388} & HRI & $(1.26 \pm 0.11) \mathrm{E}-2$ & 11274 & 0.5 & 3 \\
\hline & PSPC & $3.67 \mathrm{E}-2$ & 11639 & 1.1 & 9 \\
\hline NGC 4394 & PSPC & $<3.08 \mathrm{E}-3$ & 8495 & 7.2 & $\cdots$ \\
\hline \multirow[t]{2}{*}{ NGC 4395} & HRI & $<2.49 \mathrm{E}-3$ & 11353 & 0.5 & $\ldots$ \\
\hline & PSPC & $(8.22 \pm 0.69) \mathrm{E}-3$ & 17038 & 1.2 & 5 \\
\hline \multirow[t]{2}{*}{ NGC 4438} & HRI & $(1.21 \pm 0.07) \mathrm{E}-2$ & 21651 & 2.0 & 4 \\
\hline & PSPC & $(6.10 \pm 0.23) \mathrm{E}-2$ & 11639 & 35.6 & 19 \\
\hline NGC 4450 & PSPC & $(9.32 \pm 0.25) \mathrm{E}-2$ & 15307 & 1.7 & 4 \\
\hline
\end{tabular}


Table 2-Continued

\begin{tabular}{|c|c|c|c|c|c|}
\hline Galaxy & Instrument & Count rate ${ }^{\mathrm{a}}$ & Exp. (s) & Off-axis angle $\left({ }^{\prime}\right)$ & $\Delta_{\mathrm{OX}}\left({ }^{\prime \prime}\right)$ \\
\hline \multirow[t]{2}{*}{ NGC 4472} & HRI & $(1.84 \pm 0.02) \mathrm{E}-1$ & 34423 & 0.6 & 4 \\
\hline & PSPC & $(2.37 \pm 0.03) \mathrm{E}-1$ & 25951 & 1.0 & 7 \\
\hline NGC 4477 & PSPC & $(3.74 \pm 0.23) \mathrm{E}-2$ & 7351 & 10.3 & 15 \\
\hline \multirow[t]{2}{*}{ NGC 4486} & HRI & $1.50 \pm 0.01$ & 45108 & 0.3 & 3 \\
\hline & PSPC & $11.86 \pm 0.02$ & 30435 & 0.3 & 4 \\
\hline NGC 4494 & PSPC & $(2.34 \pm 0.14) \mathrm{E}-2$ & 12015 & 45.3 & 13 \\
\hline NGC 4501 & HRI & $(1.16 \pm 0.11) \mathrm{E}-2$ & 10418 & 5.6 & 14 \\
\hline NGC 4548 & PSPC & $<2.01 \mathrm{E}-2$ & 1262 & 44.7 & $\cdots$ \\
\hline NGC 4550 & PSPC & $(1.43 \pm 0.09) \mathrm{E}-2$ & 16660 & 15.0 & 10 \\
\hline \multirow[t]{2}{*}{ NGC 4565} & HRI & $(1.05 \pm 0.14) \mathrm{E}-2$ & 5312 & 1.4 & 12 \\
\hline & PSPC & $(2.41 \pm 0.11) \mathrm{E}-2$ & 19707 & 1.1 & 5 \\
\hline NGC 4579 & PSPC & $(6.36 \pm 0.08) \mathrm{E}-1$ & 9313 & 0.7 & 2 \\
\hline \multirow[t]{2}{*}{ NGC 4636} & HRI & $(1.94 \pm 0.03) \mathrm{E}-1$ & 22050 & 0.2 & 10 \\
\hline & PSPC & $(8.54 \pm 0.08) \mathrm{E}-1$ & 13070 & 0.2 & 10 \\
\hline \multirow[t]{2}{*}{ NGC 4639} & HRI & $(2.08 \pm 0.16) \mathrm{E}-2$ & 8083 & 0.5 & 4 \\
\hline & PSPC & $(3.47 \pm 0.23) \mathrm{E}-2$ & 6604 & 0.5 & 7 \\
\hline \multirow[t]{2}{*}{ NGC 4651} & HRI & $(1.50 \pm 0.24) \mathrm{E}-3$ & 25396 & 2.4 & 9 \\
\hline & PSPC & $(1.28 \pm 0.11) \mathrm{E}-2$ & 10481 & 1.2 & 9 \\
\hline NGC 4698 & IPC & $(7.05 \pm 0.83) \mathrm{E}-3$ & 10355 & 0.2 & $\cdots$ \\
\hline NGC 4725 & PSPC & $(6.10 \pm 0.18) \mathrm{E}-2$ & 19362 & 14.6 & 9 \\
\hline
\end{tabular}


Table 2-Continued

\begin{tabular}{|c|c|c|c|c|c|}
\hline Galaxy & Instrument & Count rate ${ }^{\mathrm{a}}$ & Exp. (s) & Off-axis angle (') & $\Delta_{\mathrm{OX}}\left({ }^{\prime \prime}\right)$ \\
\hline \multirow[t]{2}{*}{ NGC 4736} & HRI & $(9.05 \pm 0.09) \mathrm{E}-2$ & 112910 & 0.6 & 3 \\
\hline & PSPC & $(2.53 \pm 0.02) \mathrm{E}-1$ & 94819 & 0.2 & 1 \\
\hline NGC 4762 & IPC & $(1.41 \pm 0.11) \mathrm{E}-2$ & 12243 & 5.3 & $\cdots$ \\
\hline NGC 4772 & HRI & $<2.23 \mathrm{E}-3$ & 12120 & 0.6 & .. \\
\hline \multirow[t]{2}{*}{ NGC 5194} & HRI & $(3.83 \pm 0.09) \mathrm{E}-2$ & 45715 & 0.5 & 5 \\
\hline & PSPC & $(2.24 \pm 0.03) \mathrm{E}-1$ & 23956 & 0.5 & 9 \\
\hline \multirow[t]{2}{*}{ NGC 5195} & HRI & $(9.19 \pm 0.45) \mathrm{E}-3$ & 45715 & 4.4 & 9 \\
\hline & PSPC & $(3.31 \pm 0.12) \mathrm{E}-2$ & 23956 & 4.4 & 12 \\
\hline
\end{tabular}

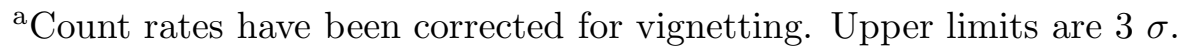


Table 3. Point-Source Flux Fractions

\begin{tabular}{ccc}
\hline \hline & \multicolumn{2}{c}{$\Sigma($ PSF $) / \Sigma($ source $)$} \\
\cline { 2 - 3 } Galaxy & PSPC & HRI \\
\hline NGC 1052 & 0.88 & 0.34 \\
NGC 1068 & 0.79 & 0.38 \\
NGC 2841 & 0.50 & $\ldots$ \\
NGC 3031 & 0.78 & 0.79 \\
NGC 3379 & $\ldots$ & 0.47 \\
NGC 3623 & 0.68 & 0.82 \\
NGC 3982 & 1.00 & $\ldots$ \\
NGC 4051 & 0.83 & 0.56 \\
NGC 4203 & 0.88 & 0.59 \\
NGC 4258 & 0.10 & 0.02 \\
NGC 4278 & 1.00 & 0.61 \\
NGC 4293 & 1.00 & $\ldots$ \\
NGC 4374 & 0.76 & 0.11 \\
NGC 4388 & 0.61 & 0.24 \\
NGC 4395 & 1.00 & $\ldots$ \\
NGC 4438 & $\ldots$ & 0.31 \\
NGC 4450 & 0.91 & $\ldots$ \\
NGC 4472 & 0.11 & 0.03 \\
NGC 4477 & 0.86 & $\ldots$ \\
NGC 448 & 0.06 & 0.03 \\
NGC 4501 & $\ldots$ & 0.26 \\
NGC 4550 & 1.00 & $\ldots$ \\
NGC 4565 & 1.00 & 1.00 \\
NGC 4579 & 1.00 & $\ldots$ \\
NGC 4636 & 0.13 & 0.03 \\
NGC 4639 & 1.00 & 0.70 \\
NGC 4651 & 1.00 & 0.82 \\
NGC 4725 & 0.83 & $\ldots$ \\
NGC 4736 & 0.47 & 0.11 \\
NGC 5194 & 0.33 & 0.16 \\
NGC 5195 & 0.25 & 0.13 \\
\hline & &
\end{tabular}


Table 4. Observed X-ray Fluxes and Luminosities

\begin{tabular}{cccccc}
\hline \hline Galaxy & $N_{\mathrm{H}}^{\mathrm{a}}$ & Model & $\chi_{\nu}^{2}$ & $F_{\mathrm{X}}{ }^{\mathrm{b}}$ & $\log L_{\mathrm{X}}^{\mathrm{c}}$ \\
\hline NGC 185 & 12.3 & $\ldots$ & $\ldots$ & $<0.534$ & $<36.83$ \\
NGC 404 & 5.31 & $\ldots$ & $\ldots$ & 0.684 & 37.87 \\
NGC 1052 & 3.07 & PL & 1.03 & 4.439 & 40.32 \\
NGC 1058 & 6.65 & $\ldots$ & $\ldots$ & $<0.347$ & $<38.77$ \\
NGC 1068 & 3.53 & PL+RS & 1.18 & 171.3 & 41.90 \\
NGC 2681 & 2.45 & $\ldots$ & $\ldots$ & $<1.056$ & $<39.53$ \\
NGC 2841 & 1.45 & PL & 1.25 & 1.970 & 39.64 \\
NGC 3031 & 4.16 & PL & 1.27 & 103.1 & 39.50 \\
NGC 3379 & 2.75 & $\ldots$ & $\ldots$ & 1.121 & 39.07 \\
NGC 3486 & 1.91 & $\ldots$ & $\ldots$ & $<0.607$ & $<38.67$ \\
NGC 3623 & 2.16 & $\ldots$ & $\ldots$ & 1.807 & 39.16 \\
NGC 3982 & 1.22 & $\ldots$ & $\ldots$ & 1.528 & 39.79 \\
NGC 4051 & 1.32 & PL+RS & 1.14 & 115.5 & 41.72 \\
NGC 4138 & 1.36 & $\ldots$ & $\ldots$ & $<1.153$ & $<39.67$ \\
NGC 4168 & 2.56 & $\ldots$ & $\ldots$ & 0.823 & 39.55 \\
NGC 4203 & 1.19 & PL & 1.28 & 21.25 & 40.45 \\
NGC 4258 & 1.16 & PL+RS & 1.07 & 2.501 & 39.22 \\
NGC 4278 & 1.77 & $\ldots$ & $\ldots$ & 5.921 & 39.91 \\
NGC 4293 & 2.58 & $\ldots$ & $\ldots$ & 0.673 & 39.48 \\
NGC 4314 & 1.78 & $\ldots$ & $\ldots$ & 1.355 & 39.27 \\
NGC 4346 & 1.13 & $\ldots$ & $\ldots$ & $<1.904$ & $<39.88$ \\
NGC 4374 & 2.60 & PL+RS & 1.28 & 13.39 & 40.76 \\
NGC 4388 & 2.60 & PL+RS & 1.25 & 3.949 & 40.24 \\
NGC 4394 & 2.52 & $\ldots$ & $\ldots$ & $<0.344$ & $<39.18$ \\
NGC 4395 & 1.35 & $\ldots$ & $\ldots$ & 0.862 & 38.20 \\
NGC 4438 & 2.66 & PL & 1.18 & 7.430 & 40.53 \\
NGC 4450 & 2.37 & PL & 1.10 & 9.839 & 40.65 \\
NGC 4472 & 1.66 & PL+RS & 1.08 & 8.179 & 40.56 \\
NGC 4477 & 2.64 & $\ldots$ & $\ldots$ & 4.237 & 40.28 \\
NGC 4486 & 2.54 & PL+RS & 1.39 & 72.12 & 41.58 \\
NGC 4494 & 1.52 & $\ldots$ & $\ldots$ & 2.564 & 39.54 \\
NGC 4501 & 2.48 & $\ldots$ & $\ldots$ & 4.019 & 40.25 \\
NGC 4548 & 2.36 & $\ldots$ & $\ldots$ & $<2.354$ & $<40.01$
\end{tabular}


Table 4-Continued

\begin{tabular}{cccccc}
\hline \hline Galaxy & $N_{\mathrm{H}}^{\mathrm{a}}$ & Model & $\chi_{\nu}^{2}$ & $F_{\mathrm{X}}{ }^{\mathrm{b}}$ & $\log L_{\mathrm{X}}{ }^{\mathrm{c}}$ \\
\hline NGC 4550 & 2.57 & $\ldots$ & $\ldots$ & 1.654 & 39.87 \\
NGC 4565 & 1.30 & PL & 0.94 & 3.224 & 39.57 \\
NGC 4579 & 2.47 & PL & 1.05 & 78.79 & 41.51 \\
NGC 4636 & 1.81 & PL+RS & 1.01 & 10.42 & 40.66 \\
NGC 4639 & 2.35 & $\ldots$ & $\ldots$ & 4.104 & 40.25 \\
NGC 4651 & 1.99 & $\ldots$ & $\ldots$ & 1.200 & 39.70 \\
NGC 4698 & 1.87 & $\ldots$ & $\ldots$ & 1.599 & 39.83 \\
NGC 4725 & 1.00 & PL & 1.00 & 3.264 & 39.91 \\
NGC 4736 & 1.44 & PL+RS & 1.11 & 9.095 & 39.49 \\
NGC 4762 & 2.04 & $\ldots$ & $\ldots$ & 3.382 & 40.14 \\
NGC 4772 & 1.79 & $\ldots$ & $\ldots$ & $<0.798$ & $<39.47$ \\
NGC 5194 & 1.57 & PL+RS & 0.96 & 6.339 & 39.76 \\
NGC 5195 & 1.56 & PL+RS & 1.35 & 0.845 & 39.00 \\
\hline
\end{tabular}

Note. - If the flux was obtained from a fitted spectrum, the type of spectral model and the reduced $\chi^{2}$ for the best fit are given, and typical errors are $\pm 10 \%$ or less. Otherwise, the flux was obtained with the count rate and the "standard" model, with uncertainties of $\pm 20 \%$ or less; see $\S 4.2$.

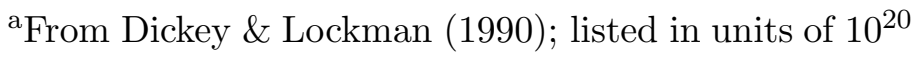
$\mathrm{cm}^{-2}$.

${ }^{\mathrm{b}} 0.1-2.4 \mathrm{keV}$ observed fluxes in units of $10^{-13} \mathrm{erg} \mathrm{s}^{-1}$ $\mathrm{cm}^{-2}$.

${ }^{\mathrm{c}} 0.1-2.4 \mathrm{keV}$ luminosities (ergs s${ }^{-1}$ ) have been corrected for Galactic absorption.

${ }^{\mathrm{d}}$ One of the eight very extended objects which received the special correction for extended emission discussed in $\S 4.1$. 
Table 5. X-ray Luminosity Statistics

\begin{tabular}{lcccc}
\hline \hline & \multicolumn{2}{c}{$\log \left(L_{\mathrm{X}} / \mathrm{ergs} \mathrm{s}^{-1}\right)$} & \multicolumn{2}{c}{$L_{\mathrm{X}} / L_{\mathrm{H} \alpha}$} \\
\cline { 2 - 5 } & Median & $\mathrm{IQR}^{\mathrm{a}}$ & Median & $\mathrm{IQR}^{\mathrm{a}}$ \\
\hline All objects & 39.66 & $39.14-40.27$ & 7.0 & $1.4-27.8$ \\
LINERs & 39.54 & $39.11-40.28$ & 7.0 & $2.1-45.1$ \\
Seyferts & 39.76 & $38.88-40.25$ & 3.0 & $0.8-15.5$ \\
Type 1 & 39.91 & $39.36-40.53$ & 3.1 & $0.9-14.1$ \\
Type 2 & 39.52 & $39.01-39.89$ & 13.4 & $1.4-28.7$ \\
\hline
\end{tabular}

${ }^{\mathrm{a}} \mathrm{IQR}=$ interquartile ratio; the range containing the middle $50 \%$ of the values. 
Fig. 1.- (a) PSPC and (b) HRI radial profiles (crosses) of the nuclear X-ray sources in several galaxies, along with the theoretical PSFs (solid lines) corresponding to the source position on the relevant detector. Below each profile, the normalized residuals (i.e., [source profile - PSF] / $[1 \sigma$ source profile uncertainty]) are plotted. To the right of the profiles are the ROSAT images of the sources, which are $12^{\prime}$ square in $(a)$ and $6^{\prime}$ square in $(b)$. Coordinates are J2000. The nucleus is centered in these images. The different plots represent the range of profiles observed in our LLAGN sample: point-like (NGC 4450 and NGC 4565), slightly extended (NGC 4725 and NGC 4051), and very extended (NGC 4258 and NGC 4736). 

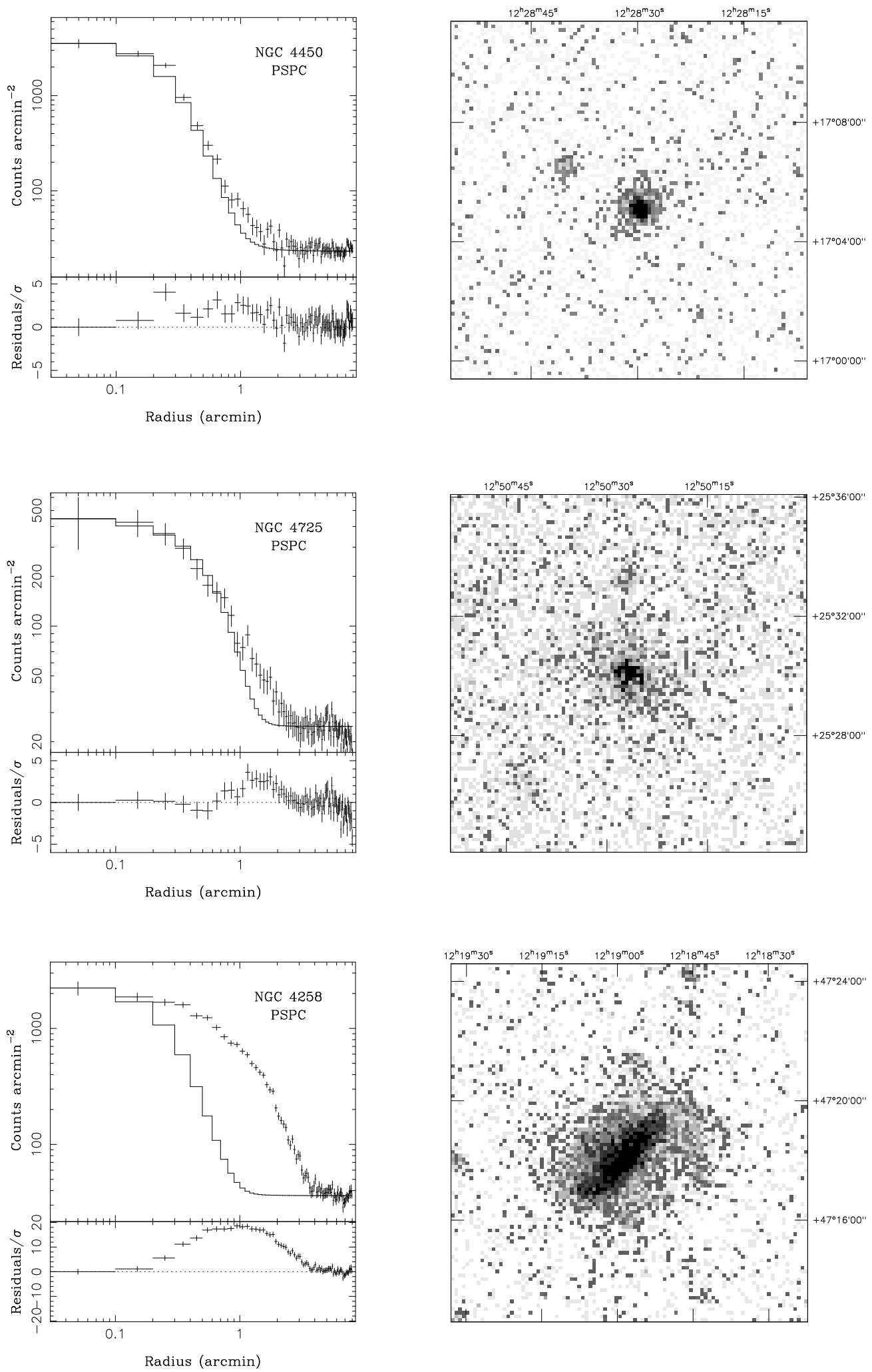

Fig. 1a 

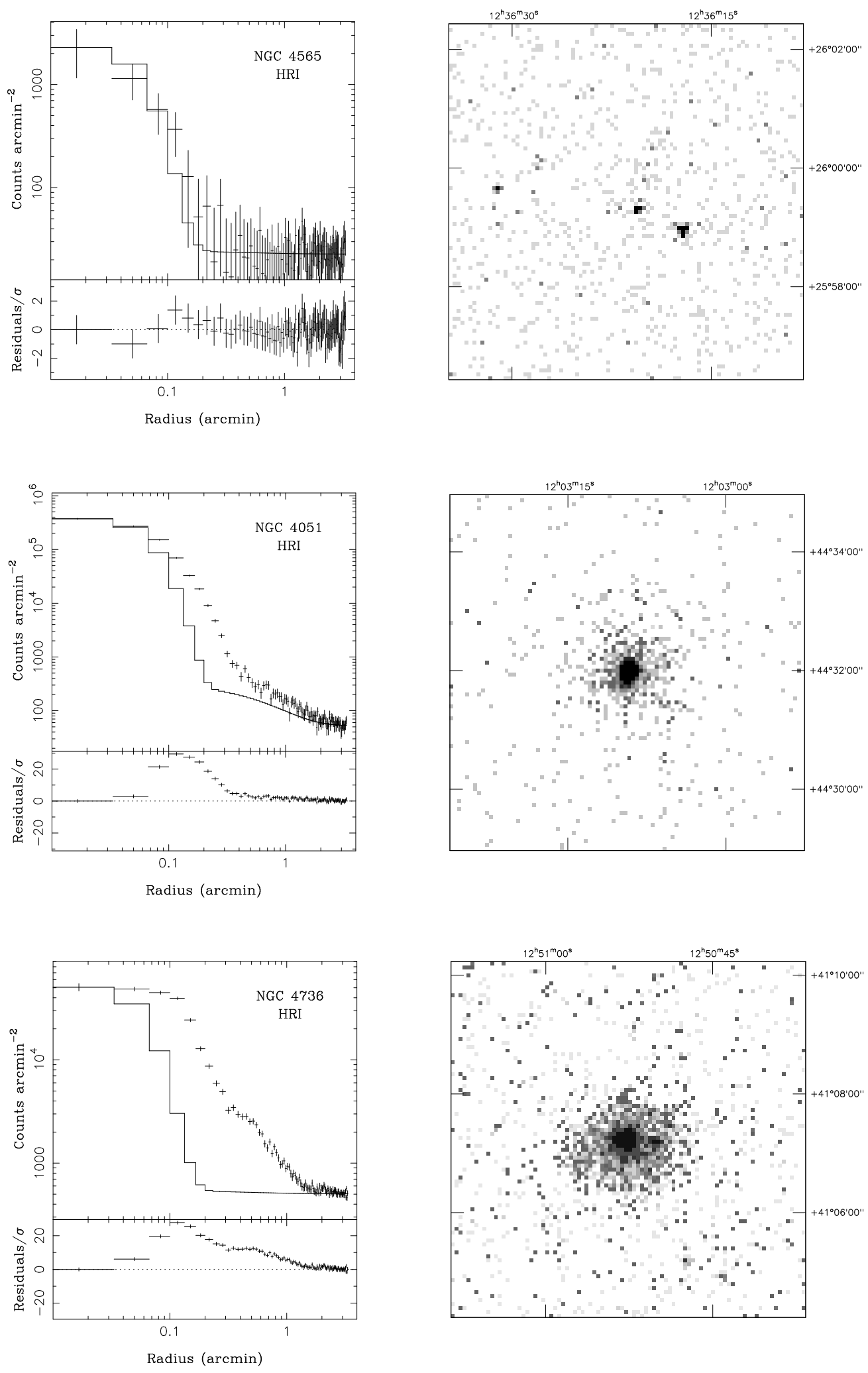

Fig. 1b 


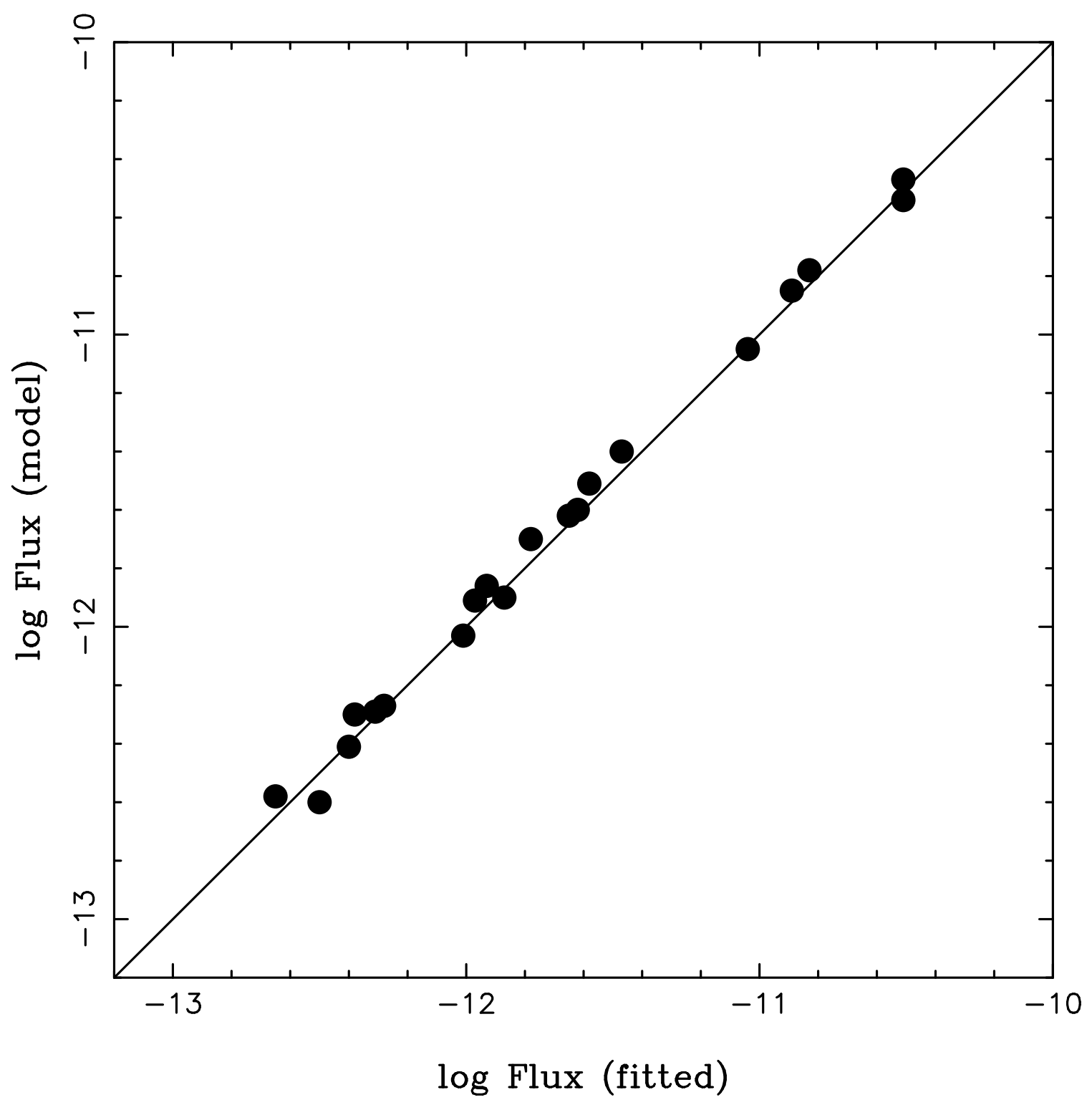

Fig. 2.- Comparison of 0.1-2.4 keV fluxes derived using the observed count rate and the "standard" spectral model with those derived from careful model fitting, for well-detected PSPC sources (i.e., those with at least 300 net source counts). The solid line indicates $F$ (model) $=F$ (fitted). The close agreement of the two flux determinations indicates that the standard model may be applied to obtain accurate fluxes for weaker sources. 


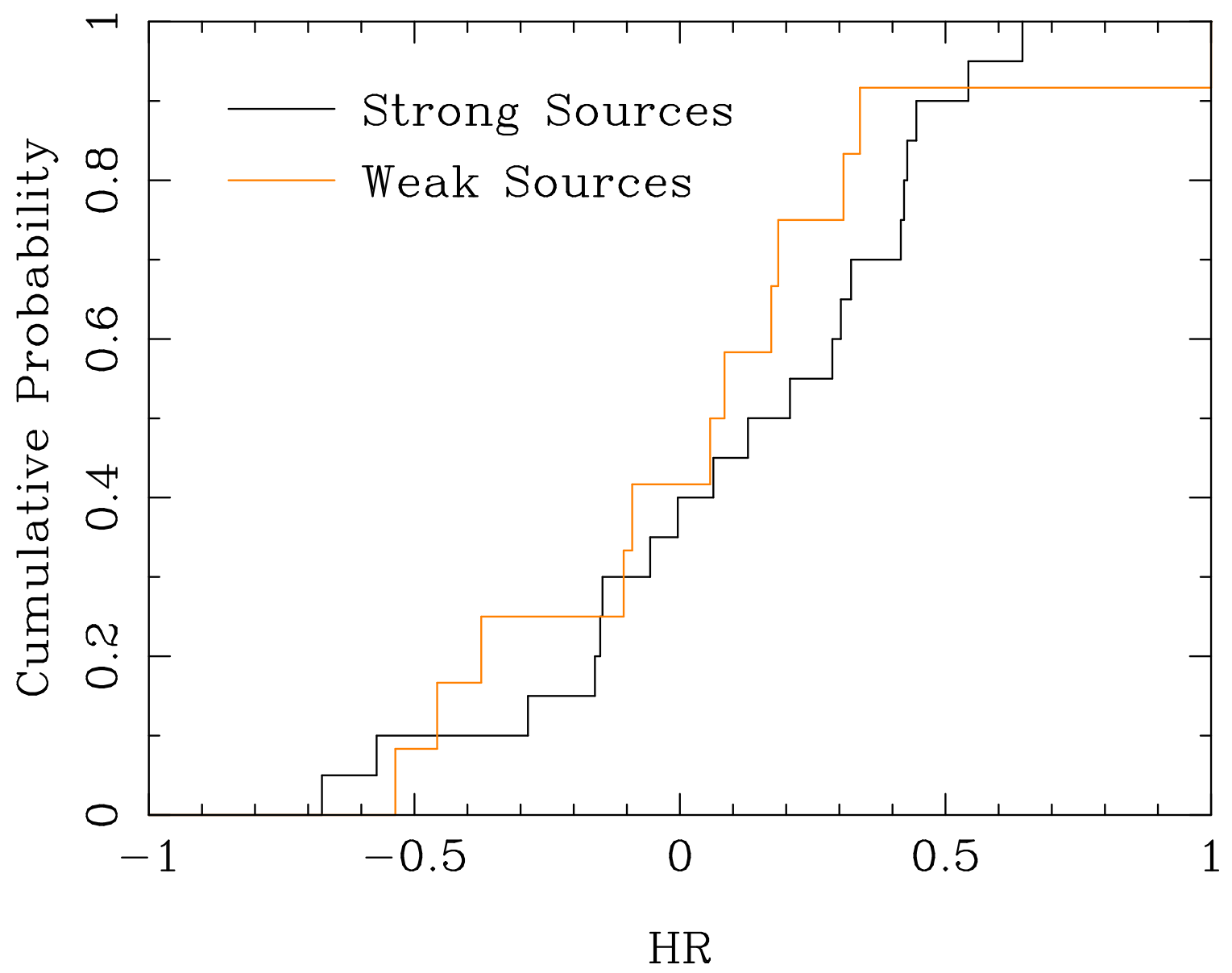

Fig. 3.- Cumulative probability distributions of the hardness ratios of strongly and weakly detected sources. The maximum value of the absolute difference between the functions is $D=0.25$. If the data sets were drawn from the same distribution, the probability that $D$ would be higher is 0.67. Thus, these distributions indicate that the spectra of the two types of sources do not differ significantly. 


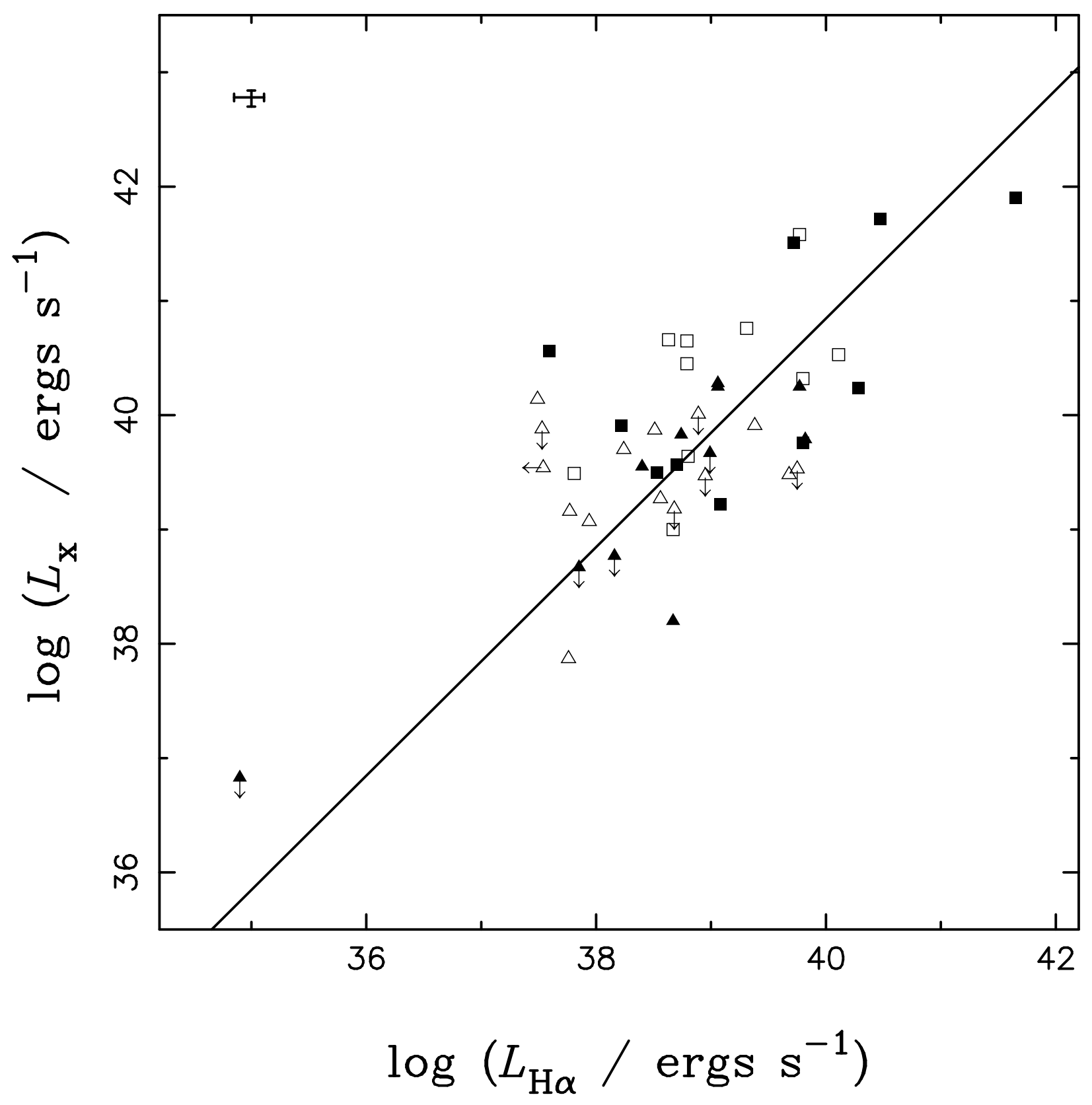

Fig. 4. $-L_{\mathrm{X}}$ vs. $L_{\mathrm{H} \alpha}$ for all objects with soft X-ray observations. The line represents the median $L_{\mathrm{X}} / L_{\mathrm{H} \alpha}$ ratio of 7 . Seyfert galaxies are represented by filled symbols, and LINERs by open symbols. Squares indicate that $L_{\mathrm{X}}$ was obtained from spectral modeling; triangles indicate that $L_{\mathrm{X}}$ was derived from the observed count rate and standard spectral model. Upper and lower limits are indicated by arrows. The error bars in the upper left corner represent a typical uncertainty in $L_{\mathrm{X}}$ of about $16 \%$ for the weaker PSPC sources, and a $30 \%$ uncertainty in $L_{\mathrm{H} \alpha}$. 

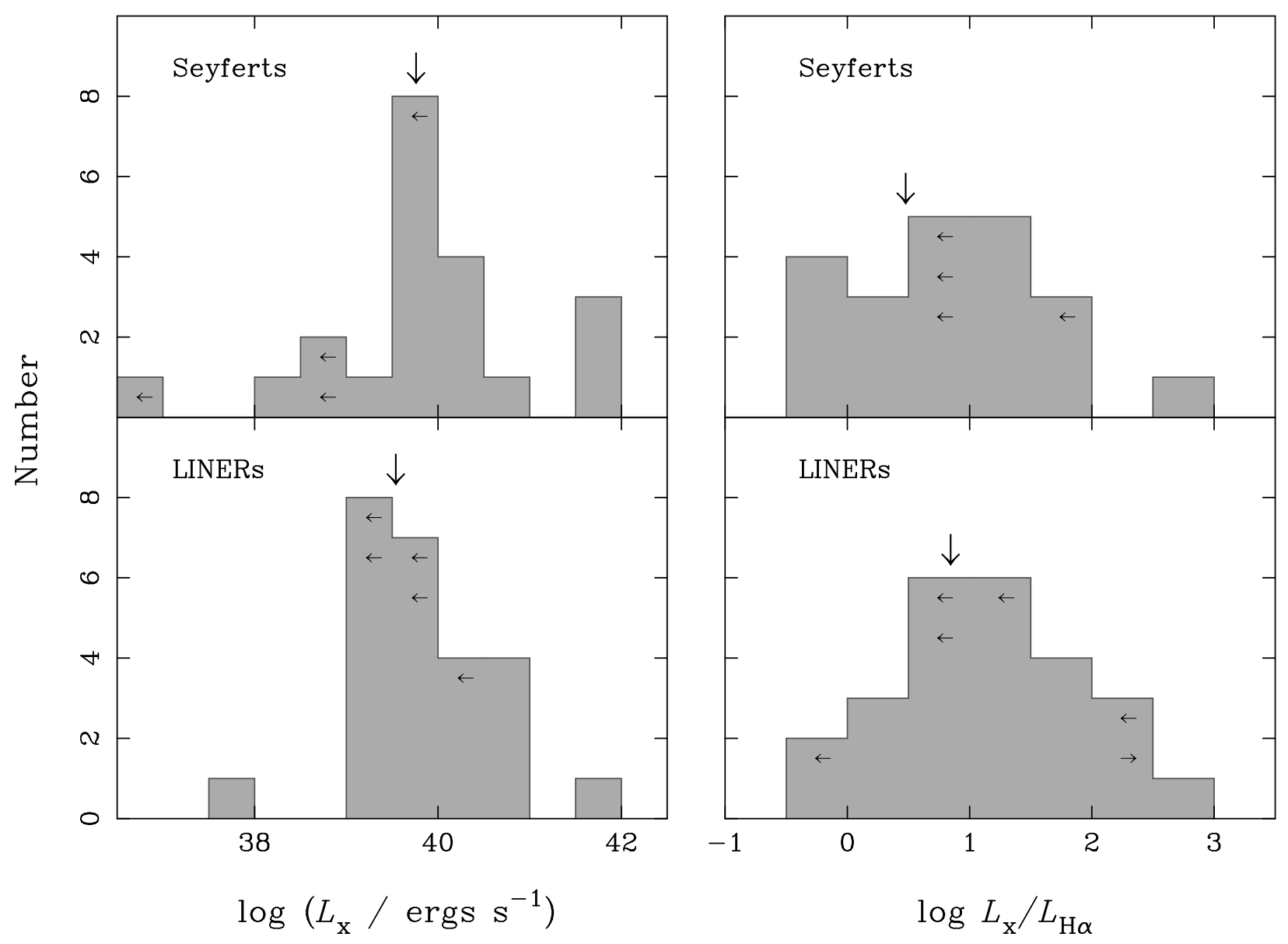

Fig. 5.- Distributions of $L_{\mathrm{X}}$ and $L_{\mathrm{X}} / L_{\mathrm{H} \alpha}$ for the Seyferts and LINERs in our sample. Median values are indicated by arrows above the histograms. 


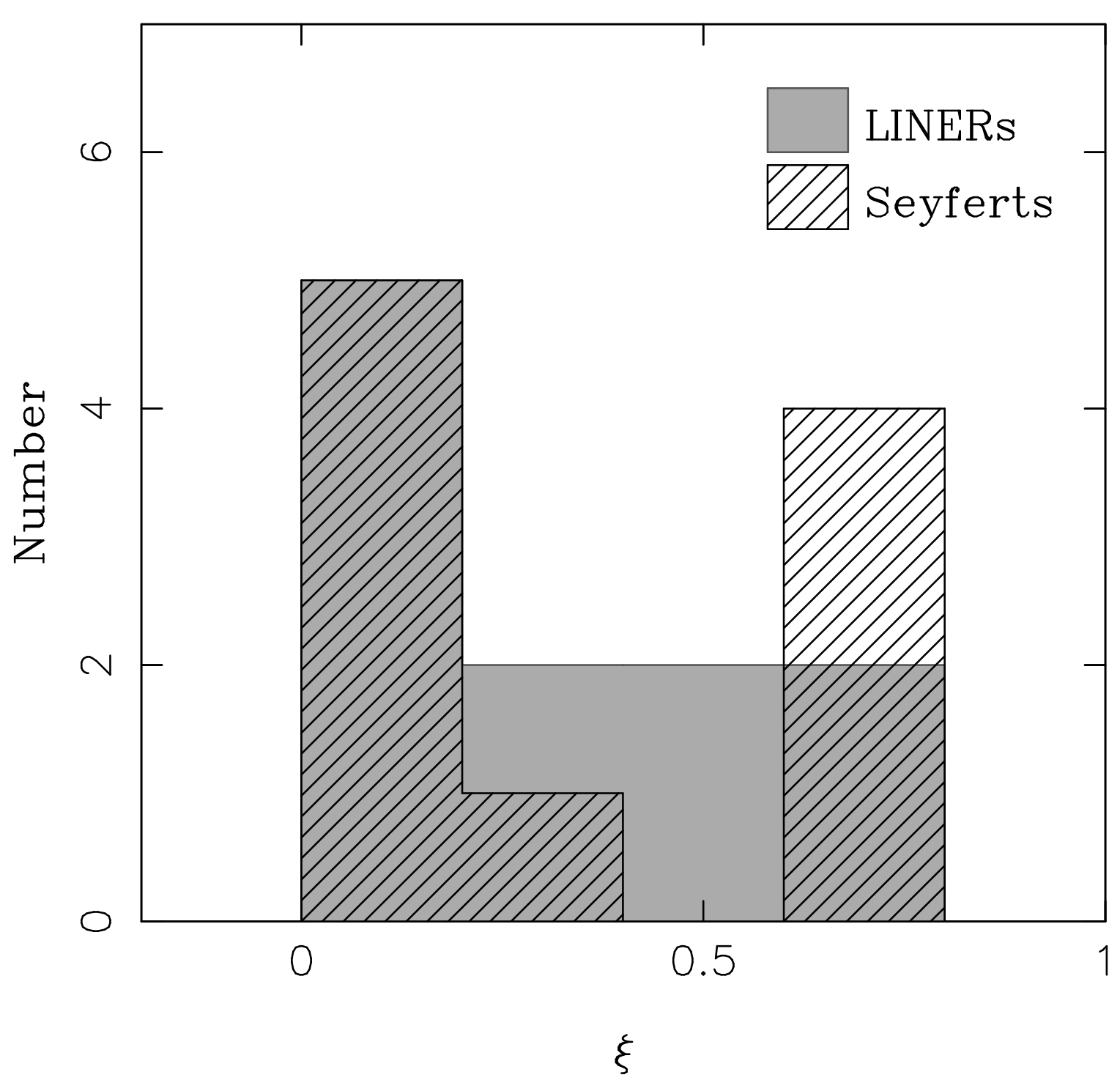

Fig. 6. - Distributions of the extended emission parameter $\xi$ for Seyferts and LINERs with measured HRI radial profiles. 

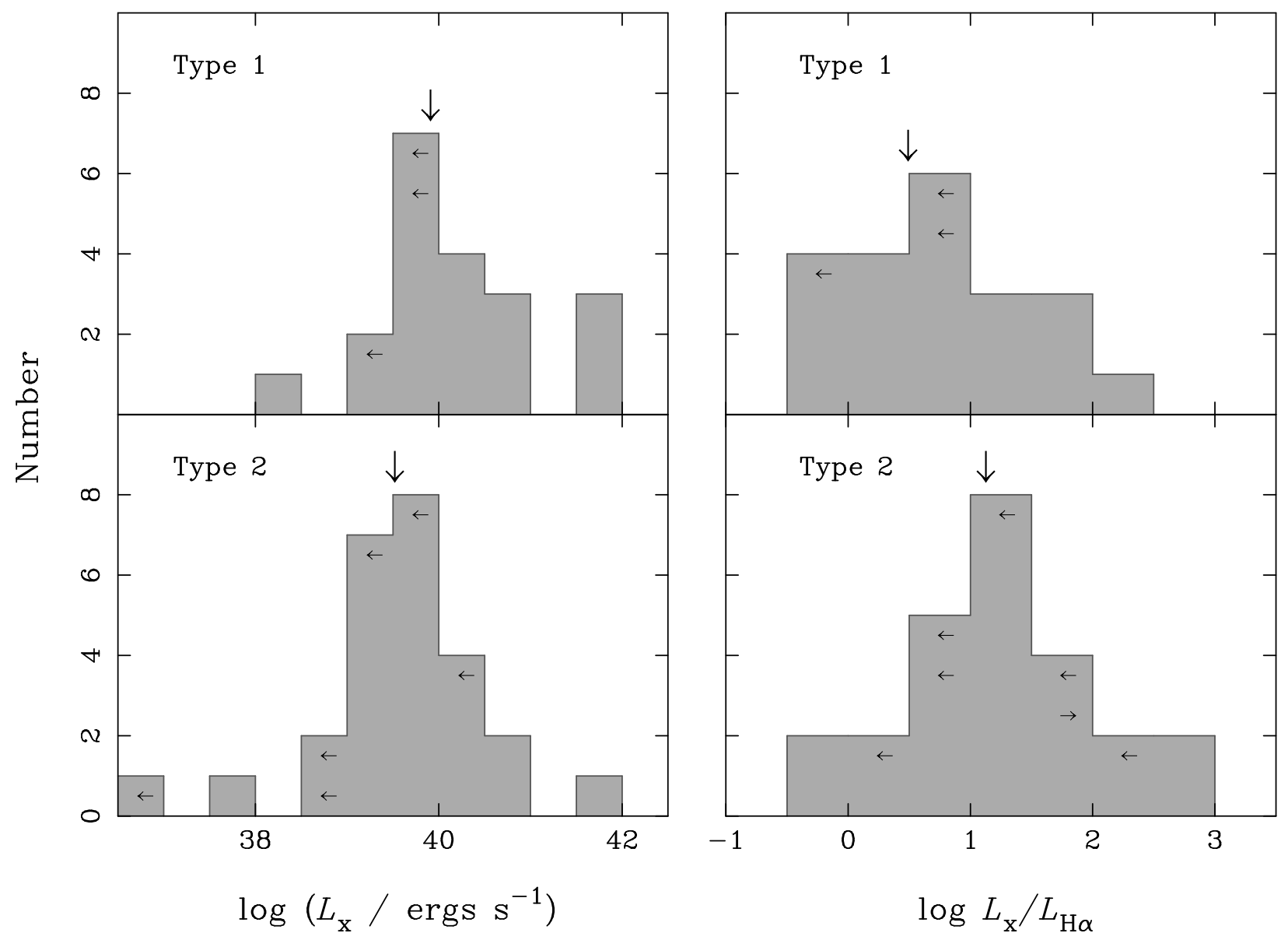

Fig. 7.- Distributions of $L_{\mathrm{X}}$ and $L_{\mathrm{X}} / L_{\mathrm{H} \alpha}$ for the type 1 and type 2 AGNs in our sample. Median values are indicated by arrows above the histograms. 